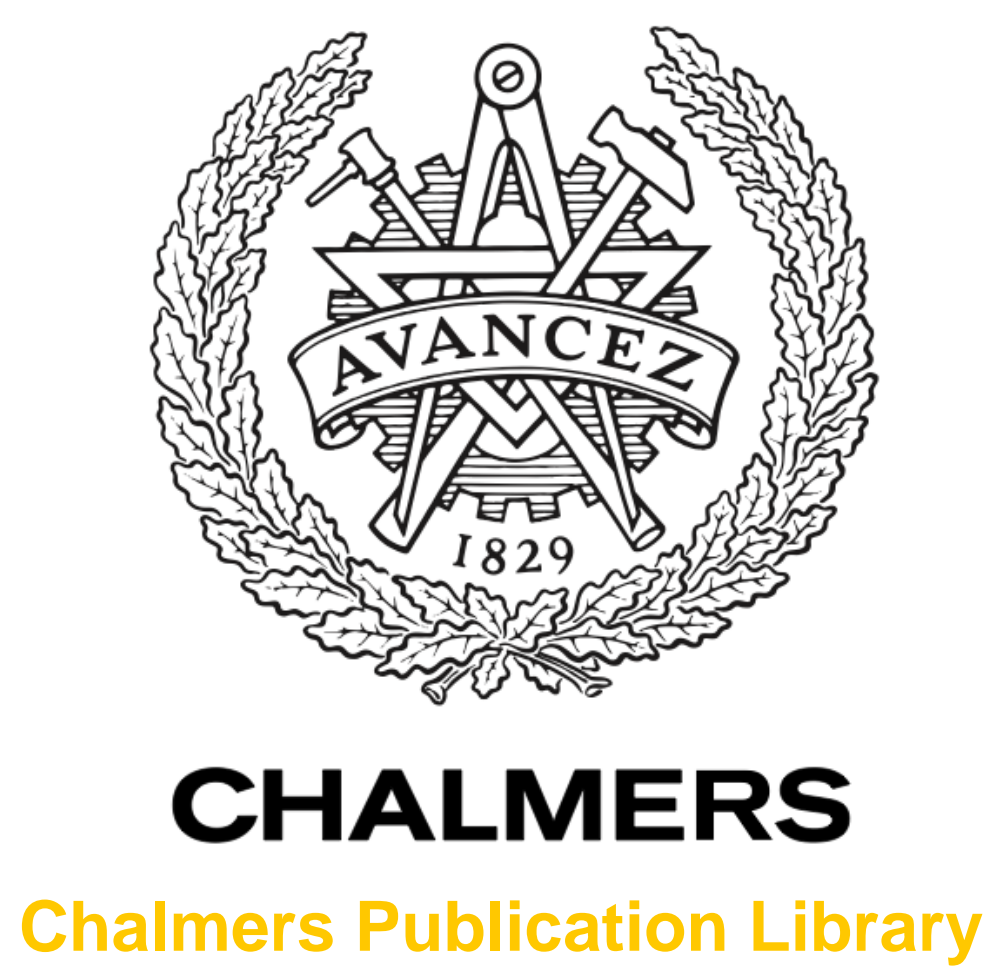

\title{
Simulation of rail roughness growth on small radius curves using a non-Hertzian and non-steady wheel-rail contact model
}

This document has been downloaded from Chalmers Publication Library (CPL). It is the author's version of a work that was accepted for publication in:

Wear, Special issue. Proc. of the 9th Conference on Contact Mechanics and Wear of Rail/Wheel Systems, SW Jiaotong Univ, State Key Lab Tract Power, Chengdu, China. Aug 2012 (ISSN: 0043-1648)

Citation for the published paper:

Torstensson, P. ; Pieringer, A. ; Nielsen, J. (2014) "Simulation of rail roughness growth on small radius curves using a non-Hertzian and non-steady wheel-rail contact model". Wear, Special issue. Proc. of the 9th Conference on Contact Mechanics and Wear of Rail/Wheel Systems, SW Jiaotong Univ, State Key Lab Tract Power, Chengdu, China. Aug 2012, vol. 314t(1/2).dp.p. 241.10253\%16/j.wear.2013.11.032

Downloaded from: http://publications.lib.chalmers.se/publication/199885

Notice: Changes introduced as a result of publishing processes such as copy-editing and formatting may not be reflected in this document. For a definitive version of this work, please refer to the published source. Please note that access to the published version might require a subscription. 


\title{
Simulation of rail roughness growth on small radius curves using a non-Hertzian and non-steady wheel-rail contact model
}

\author{
Peter T. Torstensson ${ }^{1)}{ }^{*}$, Astrid Pieringer ${ }^{2)}$ and Jens C.O. Nielsen ${ }^{1)}$ \\ 1) CHARMEC / Department of Applied Mechanics \\ 2) CHARMEC / Division of Applied Acoustics \\ Chalmers University of Technology \\ SE-412 96 Gothenburg, Sweden \\ * peter.torstensson@chalmers.se
}

\begin{abstract}
A time-domain model for the prediction of long-term growth of rail roughness (corrugation) on small radius curves is presented. Both low-frequency vehicle dynamics due to curving and high-frequency vehicle-track dynamics excited by short-wavelength rail irregularities are accounted for. The influence of non-Hertzian and non-steady effects in the wheel-rail contact model on rail wear is studied. The model features a contact detection method that accounts for wheelset yaw angle as well as surface irregularities and structural flexibilities of wheelset and rail. The development of corrugation on a small radius curve is found to be highly influenced by the wheel-rail friction coefficient. For vehicle speed $25 \mathrm{~km} / \mathrm{h}$ and friction coefficient 0.3 , predictions of long-term roughness growth on the low rail show decreasing magnitudes in the entire studied wavelength interval. For friction coefficient 0.6 , roughness growth is found at several wavelengths. The corresponding calculation for the high rail contact of the trailing wheelset indicates no roughness growth independent of friction coefficient. The importance of accounting for the phase between the calculated wear and the present rail irregularity is demonstrated.
\end{abstract}

\section{INTRODUCTION}

Rail corrugation (periodic surface irregularities at distinct wavelengths) is a problem experienced by many railway networks worldwide. According to the classification suggested in [1], short-pitch corrugation developing on the low rail on curves is referred to as "rutting". The large magnitude creep forces and relative sliding between the wheel and rail make curves particularly inclined to develop rail corrugation. Because of the high representation of small radius curves, metro systems are particularly affected. Corrugation causes a pronounced dynamic loading that leads to increased generation of noise and in severe cases even damage to track and vehicle components. To manage the problem with rail corrugation, railway networks worldwide are forced to run regular and expensive grinding procedures. For example, in 2008, the cost for rail grinding of the track network (including grinding for other purposes than corrugation removal) on the Stockholm metro was 1.2 million USD [2]. This emphasises the need for an accurate simulation model that can be applied to understand and possibly mitigate the problem.

To study corrugation growth, a combination of models for short-term dynamic vehicle-track interaction and long-term damage is required. To achieve the high-frequency dynamic excitation, either an initial roughness is modelled on the wheel and rail surfaces [3-8] or the vehicle-track system develops a self-excited oscillation, e.g. a friction induced oscillation [9-10]. In early investigations by Hempelmann et al. [3-4], a linear model was applied to study the initiation of corrugation growth on tangent track. The pinned-pinned mode, where the rail is vibrating with a wavelength equal to the double sleeper spacing, was found to be the major cause. Also, the importance of the interaction between two adjacent wheelsets mounted in a bogie was discussed. More recent work has concluded that the rail coupling between adjacent wheelsets is constituting an important wavelength-fixing mechanism to rail corrugation [5-6]. Few studies in the literature consider the numerical prediction of long-term growth of rutting corrugation [9-10]. Problems of rutting corrugation appearing in curves with radius below $200 \mathrm{~m}$ on the Stuttgart tram network in Germany were investigated in [10]. By applying a model developed in the commercial software SIMPACK, the corrugation was explained by a friction induced vibration involving the first bending mode of the leading wheelset and the P2 resonance (the vertical system resonance where vehicle unsprung mass, rails and sleepers vibrate in phase on the stiffness of the ballast) of the vehicle-track system.

Modelling the wheel-rail normal contact problem according to Hertzian theory and the tangential contact problem by an approximate model, such as Kalker's simplified steady-state theory realised through FASTSIM, implies several simplifying assumptions. For example, asymmetrical normal contact stress distributions and non-elliptical shapes of the contact area as caused by non-constant curvatures of the contacting bodies are not considered. Additionally, the time- 
variant creepages and contact geometries lead to a non-steady contact problem. In numerical studies of long-term corrugation growth, approximate contact models are often applied both for the computationally cumbersome timeintegration of the dynamic vehicle-track interaction and for the calculation of wear. In recent years, several researchers have presented models that instead use non-Hertzian and non-steady contact models for the wear calculation [11-15]. It has been shown that, both for single wavelength and broadband rail roughness excitation, these models do not predict corrugation growth. The model presented in [15] allows for simulation of the longitudinal vibration of a flexible wheelset applying a non-Hertzian and non-steady contact model in the time-integration. Corrugation growth was predicted in a wavelength interval between $3 \mathrm{~cm}$ and $10 \mathrm{~cm}$. However, it was not possible to relate the peaks in the developed rail roughness to any previously known wavelength-fixing mechanism, such as for example the pinnedpinned mode of the track. This highlights the importance of using an accurate contact model in wear calculations and confirms what was stated by Grassie in [16], "the question as to whether corrugation occurs as a result of dynamic behaviour in the contact area itself must remain open".

In the current work, the time-domain model for simulation of general three-dimensional dynamic vehicle-track interaction on a small radius railway curve presented in [17] is further developed to account for non-Hertzian and nonsteady contact and wear. The simulation model is able to simultaneously capture the low-frequency vehicle dynamics due to curving and the high-frequency (up to at least $200 \mathrm{~Hz}$ ) vehicle-track dynamics excited by surface irregularities on wheels and rails. By modelling the full vehicle, the local bending modes of the rail appearing due to the constraints imposed by adjacent wheelsets are considered. The structural flexibility of wheelsets and the track is accounted for by using the finite element method. In the wear calculations, three-dimensional non-Hertzian and non-steady wheel-rail contact is modelled based on an implementation of Kalker's variational method [18]. To calculate wear, the Archard model is applied [19]. Similar conditions of the vehicle-track system as described in [2], with corrugation developing on the low rail of a $120 \mathrm{~m}$ radius curve are considered. The influence of non-Hertzian and non-steady contact on rail wear is investigated. The functionality of the model is demonstrated in several numerical examples including excitation by a single wavelength irregularity and by broadband roughness.

\section{RAIL WEAR PREDICTION MODEL}

The methodology for simulation of long-term rail roughness growth is illustrated in the flow chart of Figure 1. The influence of the carbody on the bogie curving behaviour is accounted for by performing a pre-processing step where the

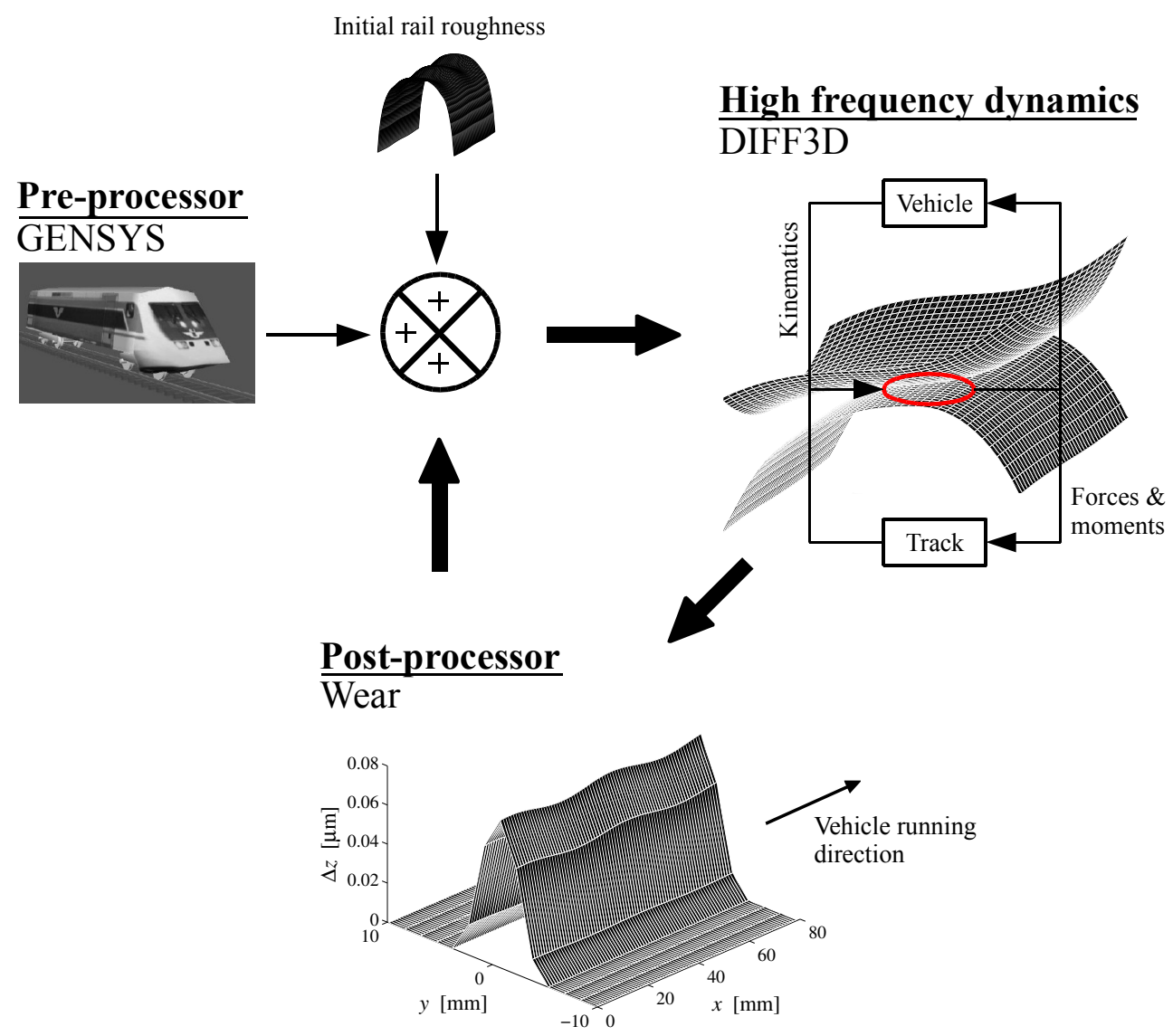

Figure 1. Illustration of the iteration scheme for simulation of long-term rail roughness growth 
dynamic interaction of a full three-unit C20 trainset and a curved track is simulated in the commercial program GENSYS [20]. Forces and moments in the secondary suspension are saved in look-up tables that are used for interpolation in the subsequent analysis. In Section 3 the in-house Matlab code DIFF3D [17] applied to simulate the high-frequency dynamic vehicle-track interaction is described. The high-frequency dynamic excitation is achieved by introducing an irregularity on the running surface of the rails, see Section 3.3. In DIFF3D, the flexible vehicle and track models interact through the wheel-rail contacts. For an input holding the positions and creepages of the contacting wheel and rail surfaces, the output from the contact model are the normal and tangential contact forces. During timeintegration in DIFF3D, the states, i.e. the displacements and velocities of the vehicle and track models, are stored in each time-step. These are used in a post-processing step to calculate rail wear for the predicted vehicle and track motion, see Section 4. In the post-process calculation of wear, the simulation model features the choice of using either Hertzian and steady-state (Hertz/FASTSIM) or non-Hertzian and non-steady-state (Kalker's exact theory) contact models. The calculated wear depth is low-pass filtered with a cut-off frequency of approximately $300 \mathrm{~Hz}$. To simulate a large number of train passages, the wear depth is extrapolated before the surface geometry is updated and used as input in the next simulation of high-frequency dynamic vehicle-track interaction. The irregularity on the rail surface is updated according to the mapping method described in Section 4.2. The procedure continues until a predefined number of train passages has been reached.

\section{VEHICLE-TRACK INTERACTION MODEL}

In the following, the model for high-frequency dynamic vehicle-track interaction DIFF3D is presented, see Figure 1. A more detailed description is given in [17].

\subsection{Track model}

The dynamic behaviour of a finite length section of the discretely supported track is accounted for by a model based on the finite element method. The model includes two rails, rail pads, sleepers and ballast, see Figure 2(a). The track gauge is $1435 \mathrm{~mm}$ with a gauge widening of $9 \mathrm{~mm}$ applied in the curve. A track length of 70 sleeper bays is considered. This ensures negligible influence of the clamped boundary conditions at the rail ends on the dynamic track response at the mid-section of the model. The rails are modelled using Euler-Bernoulli-Saint-Venant beam elements positioned at the centre-of-gravity axis of the rails. In this context, these elements are valid for frequencies below $500 \mathrm{~Hz}$ and $200 \mathrm{~Hz}$ in the vertical and lateral directions, respectively [21-22]. In order for the element passing frequency (due to the wheels passing over the elements at a given speed) to be outside of the frequency range of interest, 20 beam elements are used in each sleeper bay. The rail pads and ballast are modelled by linear springs and viscous dampers coupled in parallel. The coupling between the high and low rails through the sleepers is not modelled and the sleepers have only degrees-offreedom in vertical and lateral translations. Moreover, the longitudinal displacements of the rail elements are constrained to zero. The track is taken as symmetric with respect to the track centre line, and repetitive support properties along the rails are assumed.

The equations of motion for the track model are written as

$$
\mathbf{M}_{\mathrm{t}} \ddot{\mathbf{u}}_{\mathrm{t}}+\mathbf{C}_{\mathrm{t}} \dot{\mathbf{u}}_{\mathrm{t}}+\mathbf{K}_{\mathrm{t}} \mathbf{u}_{\mathrm{t}}=\mathbf{Q}_{\mathrm{t}}
$$

where $\mathbf{M}_{\mathrm{t}}, \mathbf{C}_{\mathrm{t}}$, and $\mathbf{K}_{\mathrm{t}}$ are the symmetric mass, damping and stiffness matrices of the track, $\mathbf{Q}_{\mathrm{t}}$ is the external load vector and $\mathbf{u}_{\mathrm{t}}$ is the corresponding track displacement vector. Due to the distribution of damping, the $N_{\mathrm{t}}$ second-order differential equations in Equation (1) are transformed into $2 N_{\mathrm{t}}$ first-order equations as

$$
\begin{gathered}
\dot{\mathbf{z}}_{\mathrm{t}}=\mathbf{A}_{\mathrm{t}} \mathbf{z}_{\mathrm{t}}+\mathbf{B}_{\mathrm{t}} \mathbf{Q}_{\mathrm{t}} \\
\mathbf{z}_{\mathrm{t}}=\left[\begin{array}{c}
\mathbf{u} \\
\dot{\mathbf{u}}_{\mathrm{t}}
\end{array}\right], \quad \mathbf{A}_{\mathrm{t}}=-\left[\begin{array}{cc}
\mathbf{0} & -\mathbf{I} \\
\mathbf{M}_{\mathrm{t}}^{-1} \mathbf{K}_{\mathrm{t}} & \mathbf{M}_{\mathrm{t}}^{-1} \mathbf{C}_{\mathrm{t}}
\end{array}\right], \quad \mathbf{B}_{\mathrm{t}}=\left[\begin{array}{c}
\mathbf{0} \\
\mathbf{M}_{\mathrm{t}}^{-1}
\end{array}\right]
\end{gathered}
$$

Through a modal expansion, using a truncated set of complex-conjugated mode pairs included in the matrix $\underline{\Lambda}_{\mathrm{t}}$, the coupled equations of motion in Equation (2) can be fully decoupled. This means, Equation (2) can be put in diagonal form suitable for time-stepping ordinary differential equation solvers as

$$
\dot{\mathbf{q}}_{\mathrm{t}}=\operatorname{diag}(\underline{a}) \mathbf{q}_{\mathrm{t}}+\Delta_{\mathrm{t}}^{\mathrm{T}} \mathbf{B}_{\mathrm{t}} \mathbf{Q}_{\mathrm{t}}
$$

where $\mathbf{q}_{\mathrm{t}}$ and $\dot{\mathbf{q}}_{\mathrm{t}}$ are the complex-valued modal coordinates and velocities, respectively, and $\operatorname{diag}(\underline{a})$ holds the complexvalued modal stiffness. In the current study, eigenmodes corresponding to eigenfrequencies up to $300 \mathrm{~Hz}$ were included in the modal expansion.

The track model has been calibrated against rail receptances measured on the small radius curve on the Stockholm metro described in [2]. At the test site, the track consists of continuously welded BV50 rails $(50 \mathrm{~kg} / \mathrm{m}$ and steel grade 
(a)
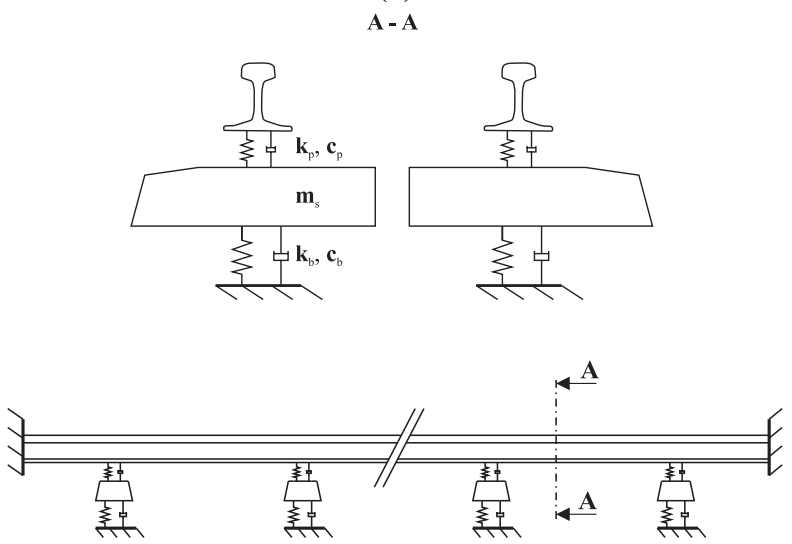

(b)

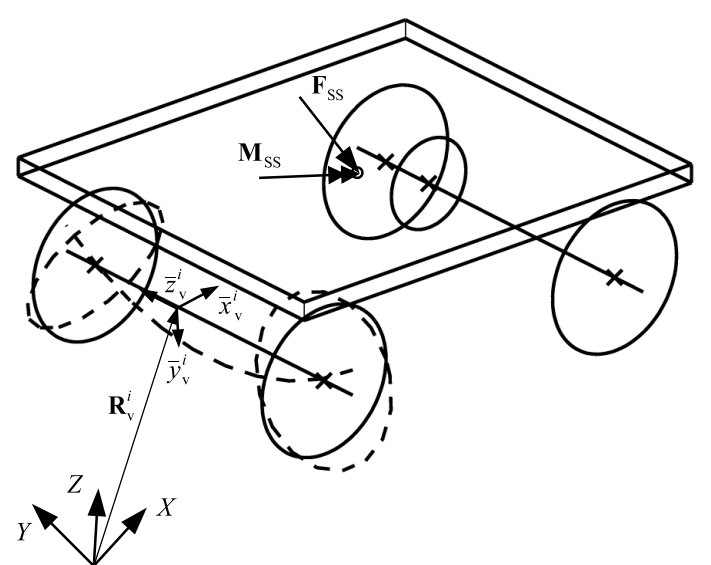

Figure 2. (a) Illustration of track model. Sleepers are modelled as rigid masses $\left(\mathbf{m}_{\mathrm{s}}\right)$. Two layers of springs and dampers, $\left(\mathbf{k}_{\mathrm{p}}, \mathbf{c}_{\mathrm{p}}\right)$ and $\left(\mathbf{k}_{\mathrm{b}}, \mathbf{c}_{\mathrm{b}}\right)$, represent the properties of rail pads and ballast, respectively. (b) Vehicle model including a rigid bogie frame and two flexible wheelsets (the driving disc is outlined only on one wheelset). Forces and moments acting in the secondary suspension are accounted for by the application of $\mathbf{F}_{\mathrm{SS}}$ and $\mathbf{M}_{\mathrm{SS}}$

R350HT) with rail inclination 1:40 on ballast subgrade. The rails are mounted, via resilient rail pads, to monobloc concrete sleepers separated by a distance of about $60 \mathrm{~cm}$. The rail receptances were measured using sledgehammer excitation, vertically on the top of the rail and laterally on the side of the rail head. Input data for the track model based on the calibration is found in the Appendix.

\subsection{Vehicle model}

The traffic on the curve described in [2] is exclusively composed of C20 trains manufactured by Bombardier Transportation. Axle load approximately 12.5 tonnes, axle distance $2.3 \mathrm{~m}$ and wheel radius $0.39 \mathrm{~m}$. A model consisting of two flexible wheelsets and a rigid bogie frame has been developed to represent this vehicle, see Figure 2(b). The primary suspension is modelled by parallel non-linear springs and viscous dampers in three directions. The car body influences the curving position of the bogie by forces and moments transmitted through the secondary suspension. In [2], the excitation frequency due to the rail corrugation was found to be between $70 \mathrm{~Hz}$ and $200 \mathrm{~Hz}$. In this frequency range it is sufficient to model the carbody as a prescribed load [23]. Rigid body simulations using a full C20 train model are performed in a pre-processing step using the commercial program GENSYS [20]. The resulting time-variant forces $\mathbf{F}_{\mathrm{ss}}$ and moments $\mathbf{M}_{\mathrm{ss}}$ in the secondary suspension are saved in look-up tables and used for interpolation in the subsequent analysis in DIFF3D. In the frequency range below $200 \mathrm{~Hz}$, structural deformation of the C20 wheelset is mainly limited to the wheel axle [17]. Therefore the wheelset model comprises a flexible wheel axle, rigid wheels and a rigid driving disc. Euler-Bernoulli-Saint-Venant beam elements are used to model the wheel axle.

The adopted multibody dynamics formulation allows for bodies that are exposed to small elastic deformations but large three-dimensional translations and rotations [24]. Each body in the multibody system is assigned with a floating coordinate system $\left(\bar{x}_{\mathrm{v}}^{i} \bar{y}_{\mathrm{v}}^{i} \bar{z}_{\mathrm{v}}^{i}\right)$ that translates and rotates with the body. The configuration of each body is defined by its reference and elastic coordinates. The reference coordinates hold the global position $\mathbf{R}_{\mathrm{v}}^{i}$ and the angular rotation $\theta_{\mathrm{v}}^{i}$ (Euler angles are used in the current study) of the floating coordinate system, while the elastic coordinates $\mathbf{q}_{\mathrm{v}}^{i}$ (modal coordinates) define the elastic deformation with respect to this coordinate system.

The nonlinear equations of motion for vehicle body $i$ can be written as

$$
\mathbf{M}_{\mathrm{v}}^{i} \ddot{\mathbf{u}}_{\mathrm{v}}^{i}+\mathbf{C}_{\mathrm{v}}^{i} \dot{\mathbf{u}}_{\mathrm{v}}^{i}+\mathbf{K}_{\mathrm{v}}^{i} \mathbf{u}_{\mathrm{v}}^{i}=\left(\mathbf{Q}_{\mathrm{v}}^{i}\right)_{\mathrm{e}}+\left(\mathbf{Q}_{\mathrm{v}}^{i}\right)_{\mathrm{v}}, \quad \mathbf{u}_{\mathrm{v}}^{i}=\left[\begin{array}{lll}
\mathbf{R}_{\mathrm{v}}^{i} & \boldsymbol{\theta}_{\mathrm{v}}^{i} & \mathbf{q}_{\mathrm{v}}^{i}
\end{array}\right]^{\mathrm{T}}
$$

where $\mathbf{M}_{\mathrm{v}}^{i}, \mathbf{C}_{\mathrm{v}}^{i}, \mathbf{K}_{\mathrm{v}}^{i}$ are the mass, damping and stiffness matrices of vehicle body $i, \mathbf{u}_{\mathrm{v}}^{i}$ is the vector of independent degrees-of-freedom of the system, and $\left(\mathbf{Q}_{\mathrm{v}}^{i}\right)_{\mathrm{e}}$ is the vector of externally applied loads such as the forces in the wheel-rail contacts. The quadratic velocity vector $\left(\mathbf{Q}_{\mathrm{v}}^{i}\right)_{\mathrm{v}}$ holds the gyroscopic and Coriolis effects. The second-order differential equations of Equation (4a) are transformed into first-order form following a similar procedure as outlined by Equation (2).

\subsection{Irregular surfaces of wheel and rail}

The simulation procedure allows for general three-dimensional motion of the vehicle on the track. The locations of contact on the wheel and rail are determined by the curving conditions (e.g. vehicle speed and track geometry) and can 
not be presumed to occur for a restricted range of positions on the wheel and rail profiles. To model the geometry of the rail surface, eight-noded quadratic two-dimensional elements are used [25]. This allows for a model of general surface irregularities with an accuracy governed by the selected element size. In the current study, the element size is approximately $1.0 \mathrm{~mm}$ and $1.5 \mathrm{~mm}$ in the longitudinal and lateral directions, respectively. The modelling of the running surfaces of wheels and rails is illustrated in Figure 3. Since wheel out-of roundness is not considered here, the surface of the wheel in the circumferential direction can be described analytically. The Swedish BV50 rail profile with an inclination of 1:40 and a S1002 wheel profile are used.

In [2], the development of roughness within a grinding interval of one year was investigated by repetitively performing measurements of rail roughness. The measured data is used to calculate a roughness level spectrum as

$$
L_{\mathrm{r}}=10 \log \left(\frac{\tilde{r}^{2}}{r_{\text {ref }}^{2}}\right)
$$

where $L_{\mathrm{r}}$ is defined in $\mathrm{dB}$ relative to the reference value $r_{\mathrm{ref}}=1 \mu \mathrm{m}$ and $\tilde{r}$ is the root mean square value of the roughness, $r(x)$, evaluated in $1 / 3$ octave bands. In the current study, rail roughness in the approximate wavelength interval $3 \mathrm{~cm}-65 \mathrm{~cm}$ is considered. This ensures a broadband roughness excitation that comprises the range of wavelengths where corrugation was found in [2]. To transform the measured roughness level spectrum into an irregularity modelled on the rail, the procedure presented in [26] is followed. The initial rail roughness profile is calculated as

$$
r(x)=\sum_{i=1}^{M} a_{i}\left\{\sum_{j=1}^{N} \sin \left(\frac{2 \pi}{\lambda_{i j}} x+\phi_{i j}\right)\right\}
$$

where the total number of $1 / 3$ octave bands and the number of sines used in each band are given by $M$ and $N$, respectively, and $\phi_{i j}$ are the mutually independent phase angles uniformly distributed between 0 and $2 \pi$. In the current work $N=100$, and $\phi_{i j}$ are determined by a uniform random distribution. The $N$ wavelengths $\lambda_{i j}$ are determined using a constant wavenumber increment $\Delta \kappa_{i}$ calculated as

$$
\Delta \kappa_{i}=\frac{2 \pi}{N}\left(\frac{1}{\lambda_{i}^{\min }}-\frac{1}{\lambda_{i}^{\max }}\right)
$$

where $\lambda_{i}^{\min }$ and $\lambda_{i}^{\max }$ are the minimum and maximum wavelength in band $i$, respectively. The amplitude of the $N$ sines in each band is obtained as

$$
a_{i}=\sqrt{\frac{2}{N}} 10^{L_{i} / 20}
$$

where the amplitudes are given in $\mu \mathrm{m}$.

\subsection{Non-Hertzian and non-steady wheel-rail contact model}

The vehicle and track subsystems are coupled through the wheel-rail contacts. In the multi-body simulation framework, this interaction is accounted for by using force elements that respond with forces and moments (normal and tangential contact forces and spin moment) when subjected to deformation (normal deformation and creepages). In the timeintegration of the vehicle-track system, the contact is modelled by a non-linear Hertzian spring [27] in the normal direction and an implementation of Kalker's simplified theory of rolling contact FASTSIM [28] in the tangential direction. The non-Hertzian and non-steady-state contact problem is considered in the post-processing step through an implementation of Kalker's variational method [18].

The contact variables are described with reference to the coordinate system $\left(\bar{x}_{\mathrm{c}} \bar{y}_{\mathrm{c}} \bar{z}_{\mathrm{c}}\right)$ of the contact which is located at the centre-of-gravity of the rail and is moving in the rolling direction at vehicle speed $v$, see Figure 3 . It is a right-hand coordinate system with the $\bar{x}_{\mathrm{c}^{-}}$and $\bar{z}_{\mathrm{c}}$-axes pointing in the longitudinal and upwards directions, respectively. As outlined in Figure 3, a potential contact area comprising of $N_{\mathrm{c}}$ elements with side lengths $\Delta x$ and $\Delta y$ in the $\bar{x}_{\mathrm{c}^{-}}$and $\bar{y}_{\mathrm{c}^{-}}$ directions, respectively, is introduced. In the current study, quadratic elements with side lengths $1.0 \mathrm{~mm}$ are used. The centre node is rigidly attached to the origin of the contact coordinate system. The geometry of the wheel and rail surfaces are modelled by four-noded linear two-dimensional elements [25]. In each time-step in the simulation of dynamic vehicle-track interaction, the surface geometry of the wheel and rail is described with respect to the potential contact area by linear interpolation.

According to Hertz/FASTSIM, contact stresses as well as the location and orientation of the contact area are determined by the kinematics and the curvatures of the contacts on the wheel and rail surfaces. In order to enhance the computational efficiency, it is customary to treat the contact detection problem separately from the solution of the contact stresses. In the proposed model, two different contact detection algorithms can be applied. An approximate and 
computationally efficient solution is achieved through the use of so-called contact point functions (KPF) pre-calculated in the commercial simulation software GENSYS. In the KPF, the location and orientation of the contact area are assumed to be dependent only on the relative lateral displacement, $\Delta y$, between wheel and rail [20]. These calculations do not consider irregularities of wheel and rail surfaces and wheelset yaw angle. In order to obtain a more accurate location of the contact, the true three-dimensional wheel and rail contact surfaces are considered online in the simulation of dynamic vehicle-track interaction. The contact point is assumed to be located at the centre-of-gravity of the rigid penetration, $h$, of wheel and rail, see Figure 3. This allows for a solution of the contact detection problem that accounts for the influence of structural deformation of wheelset and rail, wheelset yaw angle and the geometrical shift of the contact area with respect to the centre of the wheel rotation axis due to wheel and rail surface irregularities. The procedure is restricted to cases of one-point contact. However, since it is here applied only for contacts on the rail crown (low rail contact for leading wheelset and high rail contact for trailing wheelset), this restriction does not have any important implications in the current work. In Figure 3, results calculated in the post-processing step with the nonHertzian and non-steady contact model for a wheel with radius $0.39 \mathrm{~m}$ rolling over a corrugated rail surface with wavelength $40 \mathrm{~mm}$ are presented. The geometrical shift for the case of contact at the transition between a trough and a peak of the corrugation is observed. The non-elliptical and non-Hertzian distribution of normal contact stress is also evident. In the following, the non-Hertzian and non-steady contact model is described. For a more detailed description, see [18].

\subsubsection{Normal contact}

The normal contact problem is solved to determine the size and shape of the contact area as well as the distribution of normal contact pressure, $p_{I 3}$, and local normal displacements, $u_{I 3}$. The distance, $d_{I}$, between the deformed bodies at element $I$ of the potential contact area is introduced as

$$
d_{I}=h_{I}+u_{I 3}
$$

where $h_{I}$ is the rigid penetration, i.e. the distance between the wheel and the rail in their undeformed states, and $u_{I 3}$ is the displacement difference between wheel and rail. The influences of structural flexibility and surface irregularities are accounted for by the rigid penetration. The displacement difference $u_{I 3}$ only accounts for the elastic deformation of the surface in the vicinity of the contact area.
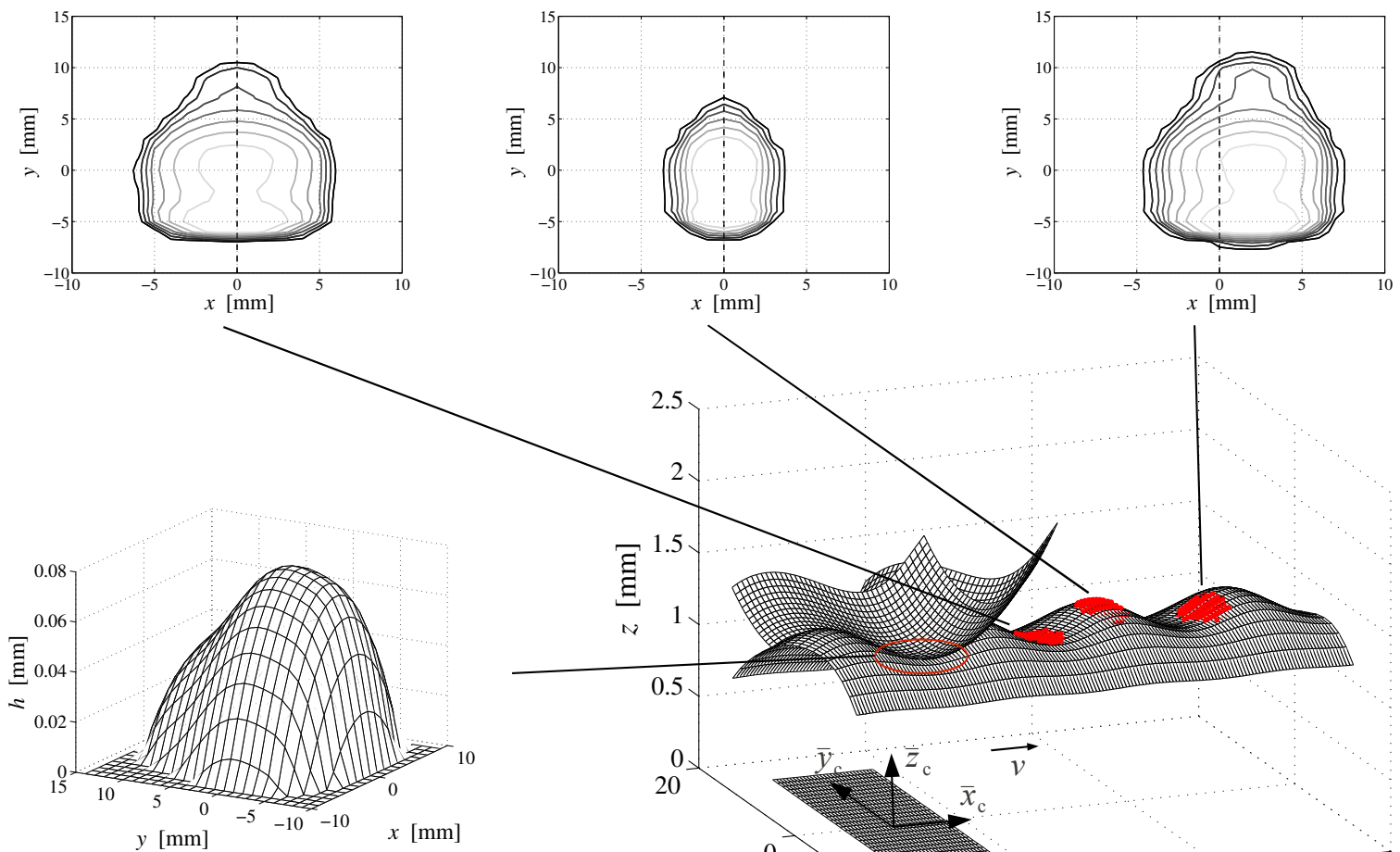
$x[\mathrm{~mm}]$

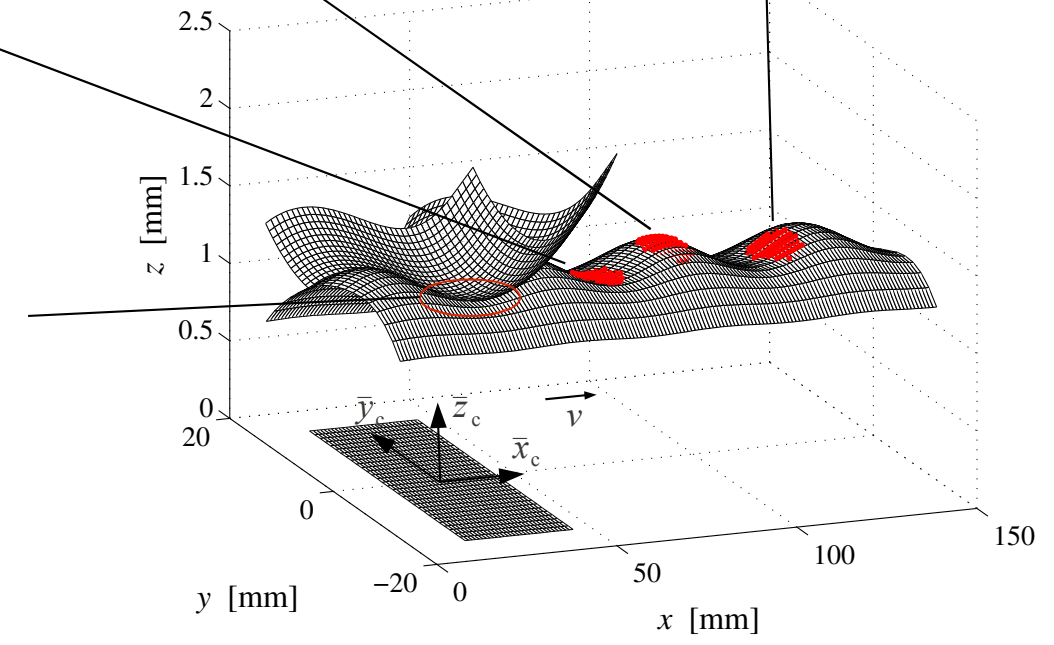

Figure 3. Illustration of a wheel rolling on a $4 \mathrm{~cm}$ single wavelength rail irregularity. The potential contact area and contact coordinate system $\left(\bar{x}_{\mathrm{c}} \bar{y}_{\mathrm{c}} \bar{z}_{\mathrm{c}}\right)$ moving at constant speed $v$ are outlined. The distribution of normal contact stress at different locations along the prescribed corrugation is shown in three contour plots. One example of the rigid penetration $h$ of the current wheel-rail contact is presented in a surface plot 
The contact conditions are formulated as

$$
\begin{aligned}
d_{I} & \geqslant 0 \\
p_{I 3} & \geqslant 0 \\
d_{I} p_{I 3} & =0
\end{aligned}
$$

If contact occurs in a surface element, the distance is zero and the normal contact pressure is positive.

\subsubsection{Tangential contact}

If contact quantities, such as the shape of the contact area or the creepages, change significantly during the passage of a particle through the contact area, the contact model needs to account for non-steady effects. For conditions similar to those in [2] (corrugation wavelength $\lambda=5 \mathrm{~cm}$ and an approximate length of the semi-axis in the rolling direction of the contact area $a=0.5 \mathrm{~cm}$ ), the $\lambda a$-ratio is equal to 10 . According to [29], non-steady analysis is necessary for $\lambda a$-ratios below 10 and hence the non-steady effects for the curving conditions in [2] may be significant.

The tangential contact problem is solved to determine the distribution of stick and slip over the contact elements, and to calculate the distribution of tangential tractions, $p_{I \tau}$, and tangential displacement difference, $u_{I_{\tau}}$. The spin creepage is estimated to have a limited influence on the contact problem due to the small contact angle at the current contact locations and is not accounted for. The local shift at element $I$ of the potential contact area, defined as the relative displacement of two opposing particles on wheel and rail during one time step $\Delta t=\Delta x / v$, is calculated as

$$
\begin{gathered}
S_{I \tau}=u_{I \tau}+W_{I \tau}^{*}-u_{I \tau}^{\prime}, \quad \tau=1,2 \\
W_{I 1}^{*}=\xi \Delta x \\
W_{I 2}^{*}=\eta \Delta x
\end{gathered}
$$

where $u_{I \tau}^{\prime}$ is the tangential displacement difference for the previous time step, and $\xi$ and $\eta$ are the longitudinal and lateral creepages, respectively. According to the terminology by Kalker [28], $W_{I \tau}$ is the rigid shift. In this work, contributions from the structural flexibility are included in the evaluation of the longitudinal and lateral creepages and hence $W_{I \tau}^{*}$ also accounts for the structural dynamics. The contact conditions within the contact area are defined as

$$
\begin{aligned}
& \text { Stick area: } \quad S_{I \tau}=0, \quad \tau=1,2, \quad \sqrt{\left(p_{I 1}^{2}+p_{I 2}^{2}\right)}<p_{I 3} \mu \\
& \text { Slip area: } \quad \frac{p_{I \tau}}{\sqrt{\left(p_{I 1}^{2}+p_{I 2}^{2}\right)}}=-\frac{S_{I \tau}}{\sqrt{\left(S_{I 1}^{2}+S_{I 2}^{2}\right)}}, \quad \quad \quad \quad=1,2, \quad \sqrt{\left(p_{I 1}^{2}+p_{I 2}^{2}\right)}=p_{I 3} \mu
\end{aligned}
$$

where $\mu$ is the friction coefficient, which is taken as constant over the contact area. Equations (13) state that the local shift vanishes in the stick area. Equation (14) ensures that slip only occurs when the tangential stress is equal to the traction bound $p_{\mathrm{I} 3} \mu$ and that traction occurs in the direction opposite to the direction of slip.

\subsubsection{Constitutive relation}

To be able to solve the normal and tangential contacts, constitutive relations relating the traction, $p_{I \tau}$, to the local displacement differences, $u_{I \tau}$, are needed. Assuming that the wheel and the rail can be locally approximated by elastic half-spaces, analytical expressions for the influence functions, $A$, are found in [28]. The traction is assumed piecewise constant for the elements of the potential contact area. The coefficient $A_{I i J j}$ gives the displacement in the $i$-direction at element $I$ due to a unit traction in the $j$-direction at element $J$. Employing the half-space approach implies important assumptions such as an elastic material response, a contact area that is small with respect to the dimensions of the contacting bodies and negligible inertial effects. For smooth wheel and rail running surfaces the latter two assumptions are approximately met for contact between wheel tread and rail crown, and in the frequency range considered in the current study.

The relation between the local displacements and stresses is given by

$$
u_{I \tau}=\sum_{\alpha}^{3} \sum_{J}^{N_{\mathrm{c}}} A_{I \tau J \alpha} p_{J \alpha}, \quad \tau=1,2,3
$$

The resulting contact forces applied as external forces on the vehicle and track sub-systems are calculated as

$$
F_{\tau}=\sum_{I}^{N_{\mathrm{c}}} p_{I \tau} \Delta x \Delta y, \quad \tau=1,2,3
$$


(a)

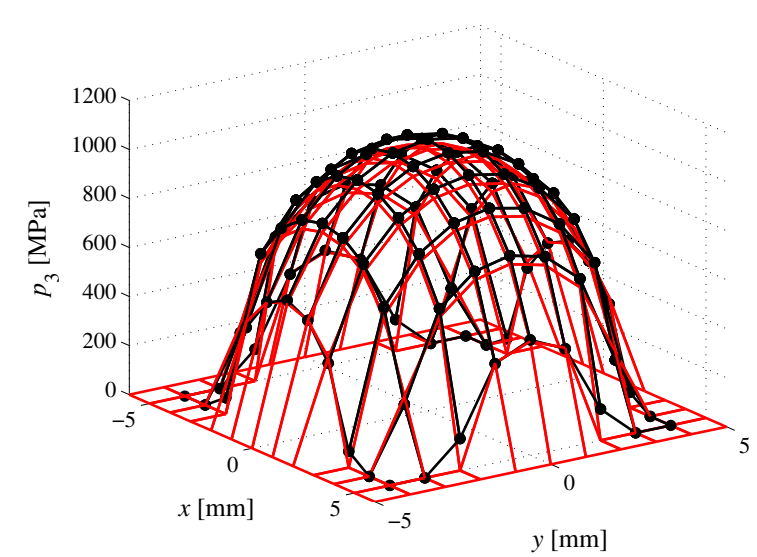

(b)

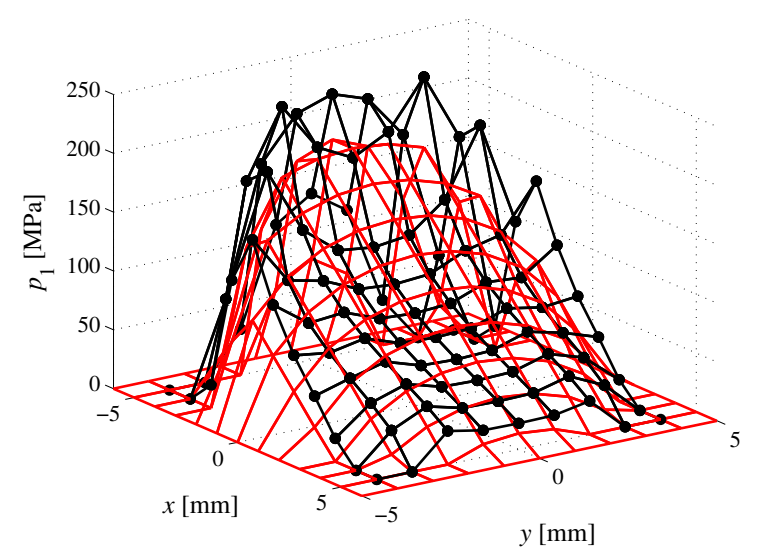

Figure 4. Comparisons of (a) normal and (b) longitudinal contact stresses calculated for the low rail contact of the leading wheelset using Hertz/FASTSIM, and the non-Hertzian and non-steady contact model. Rigid vehicle model (one bogie) travelling at $25 \mathrm{~km} / \mathrm{h}$ on a rigid tangent track. Cylindrical profiles of wheels and rails (Hertzian contact conditions). A driving torque of $500 \mathrm{Nm}$ (traction coefficient approximately 0.01 ) is applied on the wheelsets. The results calculated with FASTSIM were transformed to the same mesh as used for the non-Hertzian and non-steady contact model. $\rightarrow-$ Non-Hertzian and non-steady model, — Hertz/FASTSIM

To solve the normal and tangential contact problems, the variational method is used in combination with the active set algorithm proposed by Kalker [28].

Contact stresses calculated using Hertz/FASTSIM, or the non-Hertzian and non-steady contact model are compared in Figure 4. Hertzian contact conditions were obtained by modelling cylindrical profiles for both the wheel and rail. To create partial slip in the contact area, a driving torque of $500 \mathrm{Nm}$ was applied on the wheelsets. Good agreement is observed with regard to size and shape of the contact area. The difference in total contact force is $3 \%$ and $8 \%$ in the vertical and longitudinal directions, respectively. This serves as a form of verification of the present implementation of the non-Hertzian and non-steady contact model in the frame of DIFF3D. For a validation of the non-Hertzian and nonsteady contact model against Kalker's own implementation see [18].

\section{CALCULATION OF RAIL WEAR}

Based on experimental work, Archard and Hirst were able to identify two different wear regimes in terms of wear rate and type of wear debris: mild and severe wear [30]. Different parts of the rail profile may be subjected to different wear regimes. The wear rate at the gauge corner may be up to ten times higher than that of the rail crown [31]. In the current study, the commonly applied Archard's law for sliding wear [30] is implemented in the post-processing step, see Figure 1. Time-histories of vehicle and track states saved from the simulation in DIFF3D are used for interpolation with a fixed time-step $\Delta t=\Delta x / v$, where $v$ is the longitudinal velocity of the contact area and $\Delta x$ is the length of one contact element in the longitudinal direction. The distributions of sliding distance and normal contact pressure in the contact are either computed using Hertz/FASTSIM or the non-Hertzian and non-steady contact model described in Section 3.4.

\subsection{Wear model}

According to the Archard wear model, the volume of worn material is calculated as

$$
V_{\text {wear }}=k_{\mathrm{w}} \frac{N d}{H}
$$

where $k_{\mathrm{w}}$ is the non-dimensional wear coefficient, $N$ is the normal contact force, $d$ is the sliding distance and $H$ is the hardness of the softer material in contact. The parameter $k_{\mathrm{w}}$ is dependent on several factors, such as the normal contact pressure and the sliding velocity. For wheel and rail steels, it varies in the range $1 \cdot 10^{-4}-400 \cdot 10^{-4}[32]$. The distributions of sliding distance and normal contact pressure in the contact are either computer using Hertz/FASTSIM or the non-Hertzian and non-steady contact model. For both contact models, wear is calculated for a contact area discretised into $N_{\mathrm{c}}$ quadratic elements with side length $\Delta x=1.0 \mathrm{~mm}$. Results calculated with FASTSIM using a denser grid of elements ( 40 elements in both $x$ - and $y$-directions) are transformed to the coarser mesh of the non-steady contact model by linear interpolation. 


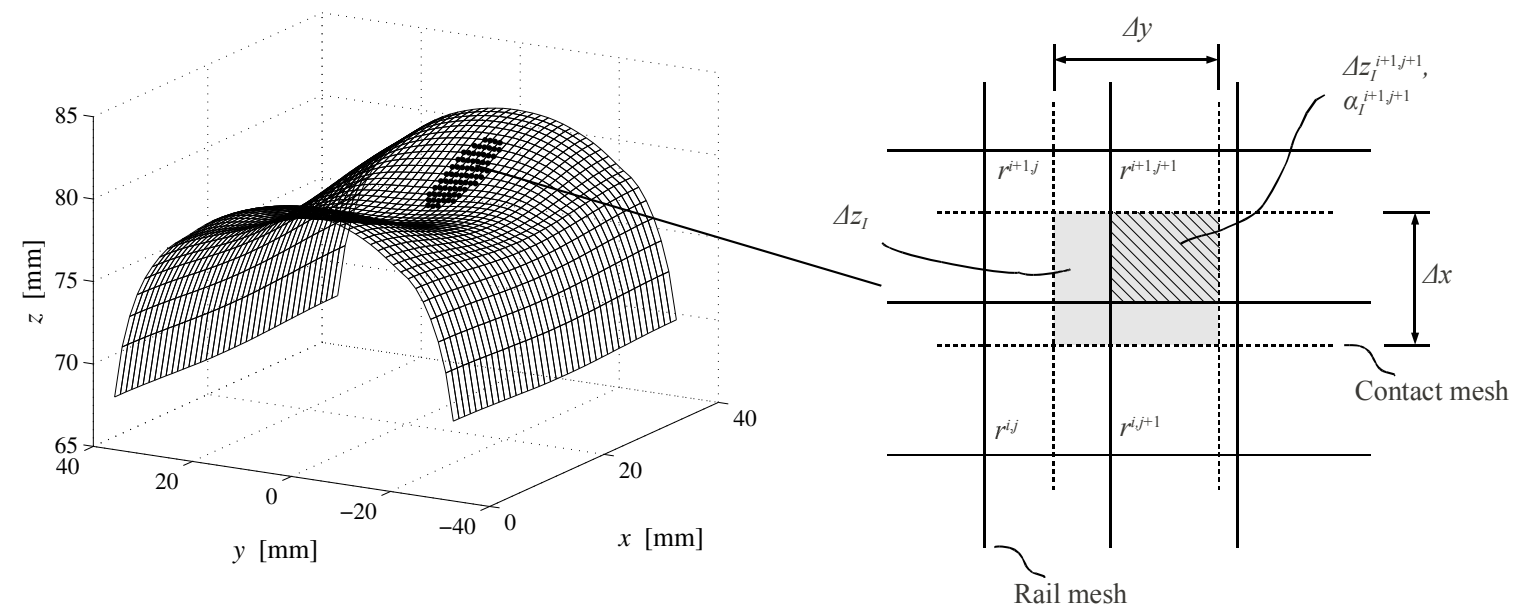

Figure 5. Illustration of procedure used for mapping of wear from the contact mesh to the rail mesh. The calculated wear depth $\Delta z_{I}$ for element $I$ of the contact mesh (coloured in grey) is distributed on several elements of the rail mesh according to area fractions. The hatched area shows the wear depth associated with rail element $r^{i+1, j+1}$

Based on Equation (17), the wear depth $\Delta z$ of one sliding element $I$ of the contact area may be calculated as

$$
\Delta z_{I}=k_{\mathrm{w}} \frac{p_{I 3} \Delta d}{H}
$$

where the sliding distance $\Delta d$ is the distance a particle on the rail slides during its passage through grid element $I$

$$
\Delta d=\mathbf{s} \Delta t
$$

Here $\mathbf{s}$ is the sliding velocity. For the non-Hertzian and non-steady contact model, the sliding distance $\Delta d$ corresponds to the Euclidean norm of the local shift $\left|S_{l}\right|$.

\subsection{Updating of the rail surface irregularity}

The contact area moves along the rail at speed approximately equal to the vehicle speed. During each time increment $\Delta t$ in the post-calculation of wear, material particles on the rail surface pass through one element of the contact mesh. However, each rail particle located along the travelling path of the contact area will pass through several contact elements during passage of a wheel. Hence, the accumulated wear during the passage of a wheel needs to be determined in order to calculate the change in rail surface shape. Previous work reported in the literature do this by different approaches. In [8], stationary contact conditions were assumed during the passage of material particles through the contact area. The accumulated wear was calculated as the sum of wear along each longitudinal strip of the discretised contact area (FASTSIM was used in this study) and assigned to the location of maximum wear. A similar procedure was used in [5]. In the current work, the calculated wear in the contact area is mapped onto the rail surface elements in each time step $\Delta t$. Figure 5 illustrates the procedure used for the calculation of the accumulated wear. The calculated wear depth $\Delta z_{I}$ for contact element $I$ (outlined in grey colour) is distributed over four elements of the rail surface mesh according to the respective area fractions. The wear depth mapped from element $I$ to rail element $r^{i+1, j+1}$ (hatched area) is calculated as

$$
\Delta z_{I}^{i+1, j+1}=\alpha_{I}^{i+1, j+1} \Delta z_{I}
$$

where $\alpha_{I}^{i+l, j+1}$ is a number between $0-1$ depending on the area fraction of element $I$ that is overlaying rail element $r^{i+1, j+1}$.

\section{NUMERICAL EXAMPLES}

The functionality of the proposed model for simulation of long-term rail roughness development will be demonstrated in several numerical examples. Conditions similar to those described in [2] with a Bombardier C20 train travelling through a curve of radius $120 \mathrm{~m}$ are considered. For these conditions, the largest magnitudes of the normal and tangential contact forces occur for the first bogie of the second car in the C20 train-set (bogie 21) [17]. In the following, results are shown only for this bogie.

The non-dimensional wear coefficient $k_{\mathrm{w}}$ and the hardness of the rail material $H$ are taken as $1 \cdot 10^{-4}$ and $3.2 \mathrm{GPa}$, 
respectively. However, the values of these parameters are here of limited importance since the purpose of this section is to demonstrate the features of the proposed model rather than to present results that quantitatively agree with field observations. The focus of the present investigation is the distribution of wear in the rolling direction. The phase shift between the calculated wear and the present rail irregularity is of major importance since it determines the potential growth of corrugation. In the following, the sum of the accumulated wear in the lateral direction is presented. This enables the phase relation between the calculated wear and the rail irregularity to be observed in two-dimensional form. Moreover, the rail irregularity is modelled with a constant magnitude in the lateral direction of the rail.

\section{$5.1 \quad$ Single wavelength initial rail irregularity}

A single wavelength irregularity with amplitude corresponding to the limit in roughness level according to ISO3095 [33] is introduced on the low rail of the curve. Results are shown for the low rail contact of the leading wheelset. The vehicle speed is $25 \mathrm{~km} / \mathrm{h}$. The calculated contact forces and wear show small amplitude oscillations at frequencies significantly exceeding the frequency range of interest in the current study $(<200 \mathrm{~Hz})$. Therefore for practical reasons the results shown in this section were low-pass filtered with a cut-off frequency of $350 \mathrm{~Hz}$ (corresponding to a wavelength of $2 \mathrm{~cm}$ at vehicle speed $25 \mathrm{~km} / \mathrm{h}$ ). Considering the potential growth of roughness, only the dynamic part of the wear depth is of interest. Therefore, the steady-state wear is subtracted from the calculated wear depth. Moreover, the wear is normalised with respect to the maximum wear depth. In Figure 6, the normalised dynamic part of the wear depth for friction coefficients 0.3 and 0.6 is shown as a function of the non-dimensional longitudinal coordinate $x / \lambda$ $(0 \leq x \leq \lambda)$. Results calculated in the post-processing step for Hertz/FASTSIM and the non-Hertzian and non-steady contact models are compared. To reduce simulation time, Hertz/FASTSIM were used to model the contact until the vehicle had reached the centre part of the flexible track model. Thereafter the non-Hertzian and non-steady contact model was switched on for a distance of approximately $3.5 \mathrm{~m}$. To exclude the transient effects caused by the change of contact models, the results in Figure 6 are taken for a wavelength in the middle of this section.

(a)
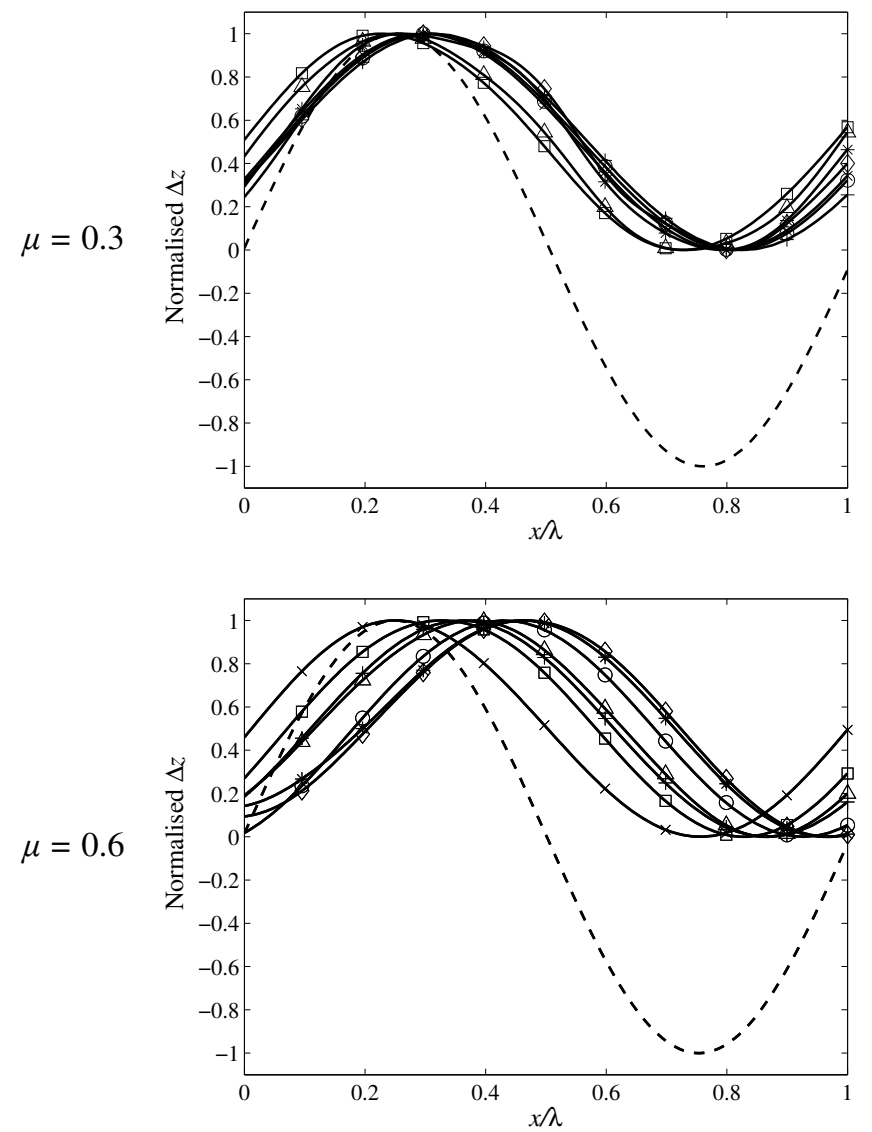

(b)
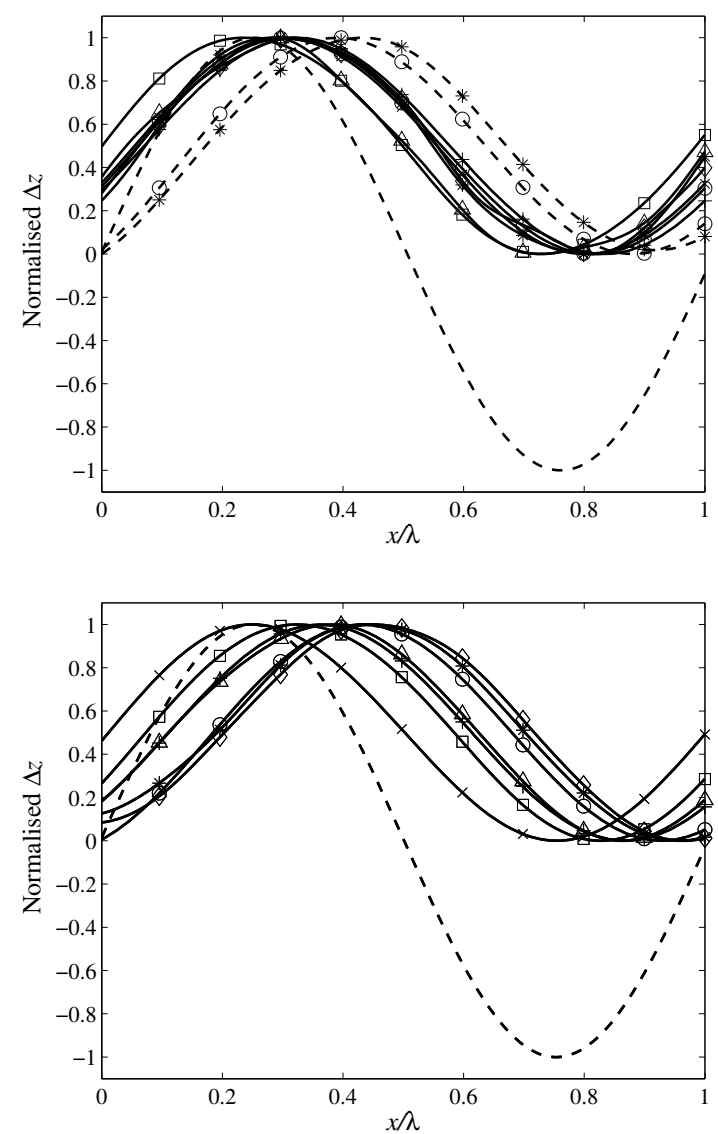

Figure 6. Normalised accumulated wear depth after one wheel passage calculated with (a) the non-Hertzian and non-steady contact model and (b) Hertz/FASTSIM using the updated location of contact. Results are shown for a vehicle travelling at $25 \mathrm{~km} / \mathrm{h}$ in a circular curve of radius $120 \mathrm{~m}$. The low rail contact of the leading wheelset and friction coefficients $\mu=0.3$ and $\mu=0.6$ are considered. An initial sinusoidal irregularity of different wavelengths (dashed line) and with wavelength-dependent amplitude corresponding to the limit according to ISO3095 [33] is modelled on the low rail. Results calculated with Hertz/FASTSIM using a pre-calculated location of contact are presented for friction coefficient 0.3 and wavelengths $5 \mathrm{~cm}$ and $9 \mathrm{~cm}$ (dashed lines with markers). 士: $4 \mathrm{~cm}, \neg: 5 \mathrm{~cm}, \rightarrow: 6 \mathrm{~cm}, \neg-7 \mathrm{~cm}, \triangle: 8 \mathrm{~cm}, \rightarrow *: 9 \mathrm{~cm}, \neg: 10 \mathrm{~cm}$ 


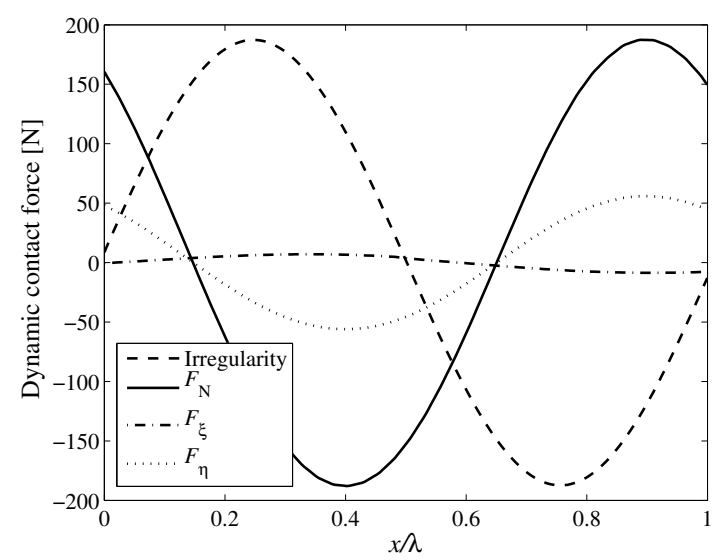

Figure 7. Time histories of dynamic wheel-rail contact forces calculated for the low rail contact of the leading wheelset during the passage of a wheel on a sinusoidal rail irregularity of $6 \mathrm{~cm}$ wavelength (outlined for reference). Amplitude of the irregularity according to ISO3095 $(2.4 \mu \mathrm{m})$ [33]. Vehicle speed $25 \mathrm{~km} / \mathrm{h}$, curve radius $120 \mathrm{~m}$ and friction coefficient $\mu=0.3$. Results were calculated using the non-Hertzian and non-steady contact model

For both friction coefficients, the results calculated using Hertz/FASTSIM (without pre-calculating contact point functions (KPF) for $\mu=0.3$ ) or the non-Hertzian and non-steady contact model are similar, see Figure 6. For friction coefficient 0.3 and for all wavelengths, the maximum wear depth occurs close to the peak of the initial irregularity (phase shift is close to zero) indicating no roughness growth. Increasing the friction coefficient to 0.6 is seen to cause a significant increase in phase between the wear and the initial rail irregularity at wavelengths $5 \mathrm{~cm}, 9 \mathrm{~cm}$ and $10 \mathrm{~cm}$. The phase shift between the calculated wear and the initial rail irregularity will lead to a translation of the rail irregularity with an increasing number of wheel passages, see Section 5.3.

The irregularity on the rail introduces a geometrical shift of the wheel-rail contact. This means that the location of contact is displaced from the position straight below the centre of the wheel axle. For the case of a single wavelength rail irregularity, the contact location is shifted towards the closest peak. As a consequence, a particle on the rail surface stays within the contact area the longest on the peaks of the rail irregularity. In particular for cases of full-slip, this leads to a contribution to the accumulated wear calculated at these positions [11]. The geometrical shift adds an additional non-linearity to the contact modelling, and it has been concluded to significantly influence the phase between the calculated wear and the present rail irregularity [5]. If the location of contact is determined based on pre-calculated contact point functions (KPF), the calculation using Hertz/FASTSIM overestimates the phase between the calculated wear and the rail irregularity. This is illustrated for two different wavelengths and friction coefficient 0.3 in Figure 6(b). As described in Section 3.4, the proposed model enables the location of contact to be updated "online" in the timeintegration of the vehicle-track interaction. This procedure does not only account for the longitudinal shift of the contact point due to the rail irregularity, but also for the influence of for example the structural flexibility of the wheelset and rail. Hence, the increased phase shift observed in Figure 6 is not only attributed to the geometrical shift.

In Figure 7, the phase relations between the contact forces and the initial rail irregularity are shown for friction coefficient 0.3 and an irregularity wavelength of $6 \mathrm{~cm}$. Similar results were obtained using Hertz/FASTSIM or the nonHertzian and non-steady contact model (only results for the non-Hertzian and non-s

teady contact model are shown here). Full slip was observed in the wheel-rail contact. The normal contact force, $F_{\mathrm{N}}$, and lateral contact force, $F_{\eta}$, lead the initial rail irregularity. The low magnitude of the longitudinal contact force, $F_{\xi}$, developed for the low rail contact of the leading wheelset is noted. No simple relationship between the phase of the contact forces and the wear depth with respect to the initial rail irregularity is observed.

\subsection{Broadband initial rail irregularity}

To obtain more realistic wheel-rail contact conditions, a broadband roughness with magnitudes corresponding to the limit according to ISO3095 is modelled on the low rail. The initial rail irregularity includes wavelength components in the approximate interval $3 \mathrm{~cm}-63 \mathrm{~cm}$ and is generated using the procedure in Section 3.3.

In order to determine the influence of the vehicle-track system on rail wear, the non-dimensional transfer function between the calculated wear depth and the rail irregularity is used

$$
\bar{H}(1 / \lambda)=\frac{\Delta \bar{Z}(1 / \lambda)}{\bar{R}(1 / \lambda)}
$$

where $\Delta \bar{Z}$ and $\bar{R}$ are the complex-valued discrete Fourier transforms of the calculated wear depth and the present rail 
irregularity, respectively. In the following, the transfer function it is applied for conditions (e.g. vehicle speed, friction, rail roughness, etc.) similar to the curve of Stockholm public transport described in [2].

For friction coefficient 0.3 and vehicle speed $25 \mathrm{~km} / \mathrm{h}$, the influence of non-Hertzian and non-steady effects are investigated for the low rail contact of the leading wheelset. The results in Figure 8 are based on a distance of $14 \mathrm{~m}$ on the mid section of the flexible track model. Results calculated for Hertz/FASTSIM, with or without an updated location of contact, and the non-Hertzian and non-steady contact model are compared with regard to the magnitude of the transfer function $\bar{H}$ evaluated in 1/24 octave bands. For longer wavelengths, similar magnitudes of the transfer function are calculated with all contact models, see Figure 8(a). For shorter wavelengths, the wear depth magnitudes calculated for the non-Hertzian and non-steady contact model exceed those obtained using Hertz/FASTSIM. The associated phase of the transfer function is shown in Figure 8(b). In the studied wavelength interval, the phase calculated with the nonHertzian and non-steady contact model, and Hertz/FASTSIM with an updated location of contact, are similar. The observation from Figure 6(b) regarding the increase in phase for Hertz/FASTSIM when the location of contact is not updated is confirmed for broadband excitation, see Figure 8(b). Especially at wavelengths below approximately $3.8 \mathrm{~cm}$, the phase calculated using Hertz/FASTSIM without updating the location of contact is significantly overestimated. The wavelength $3.8 \mathrm{~cm}$ corresponds to about three times the longitudinal length of the contact area. Updating the location of contact is observed to influence the phase between the wear depth and the rail irregularity also at wavelengths that are long in relation to the length of the contact area, see the dip at about wavelength $110 \mathrm{~mm}$ in Figure 8(b).

One important characteristic of rutting corrugation is that it only develops on the low rail in curves. When a C20 vehicle

(a)

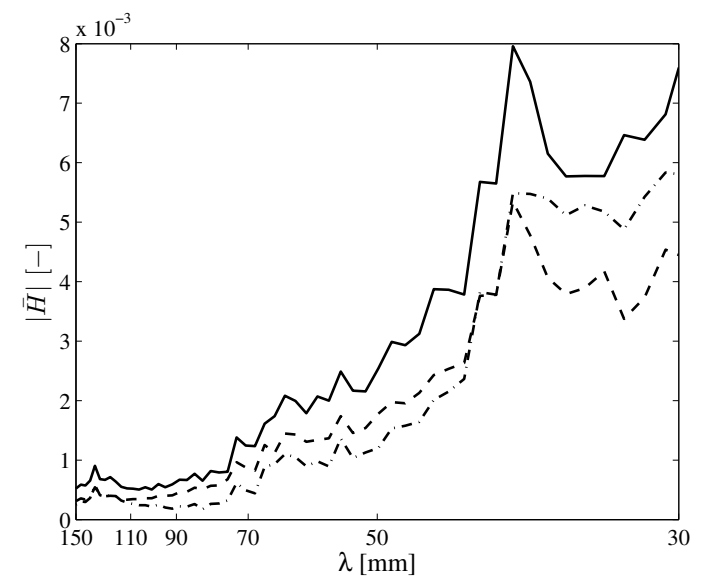

(b)

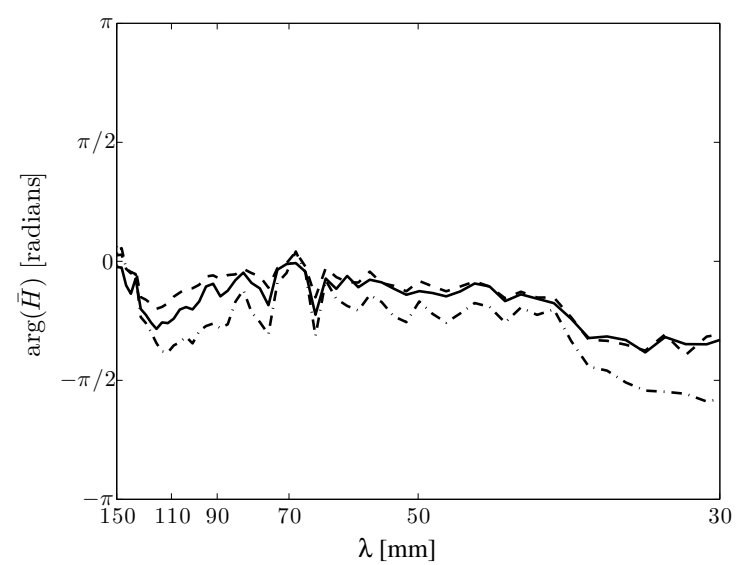

Figure 8. (a) Magnitude and (b) phase (1/24 octave bands) of the transfer function between the accumulated wear depth and the initial rail irregularity after one wheel passage of the leading wheelset on the low rail. Rail roughness modelled on the low rail with magnitude according to the limit in ISO3095 [33]. Curve radius $120 \mathrm{~m}$, vehicle speed $25 \mathrm{~km} / \mathrm{h}$ and friction coefficient 0.3 . -: Non-Hertzian and non-steady, - - : Hertz/FASTSIM, updated location of contact, - - : Hertz/FASTSIM

(a)

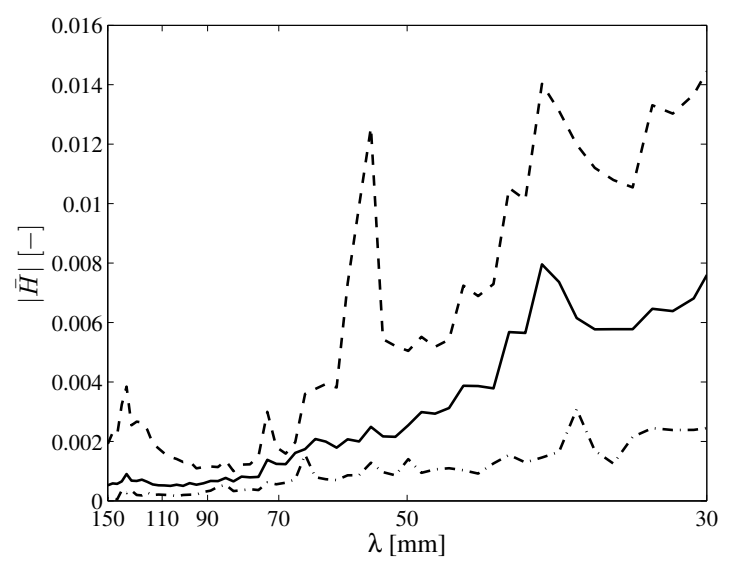

(b)

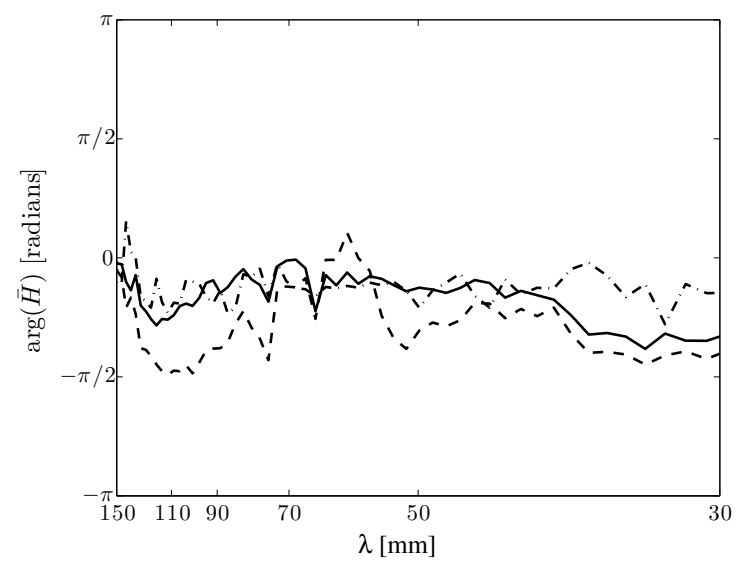

Figure 9. (a) Magnitude and (b) phase (1/24 octave bands) of the transfer function between the accumulated wear depth and the initial rail irregularity after one wheel passage of the leading wheelset on the low rail. All results calculated using the non-Hertzian and nonsteady contact model. Rail roughness modelled with magnitude according to the limit in ISO3095 [33]. Curve radius $120 \mathrm{~m}$ and vehicle speed $25 \mathrm{~km} / \mathrm{h}$. - : Low rail contact of the leading wheelset, $\mu=0.3,--:$ Low rail contact of the leading wheelset, $\mu=0.6$, - High rail contact of the trailing wheelset, $\mu=0.6$ 


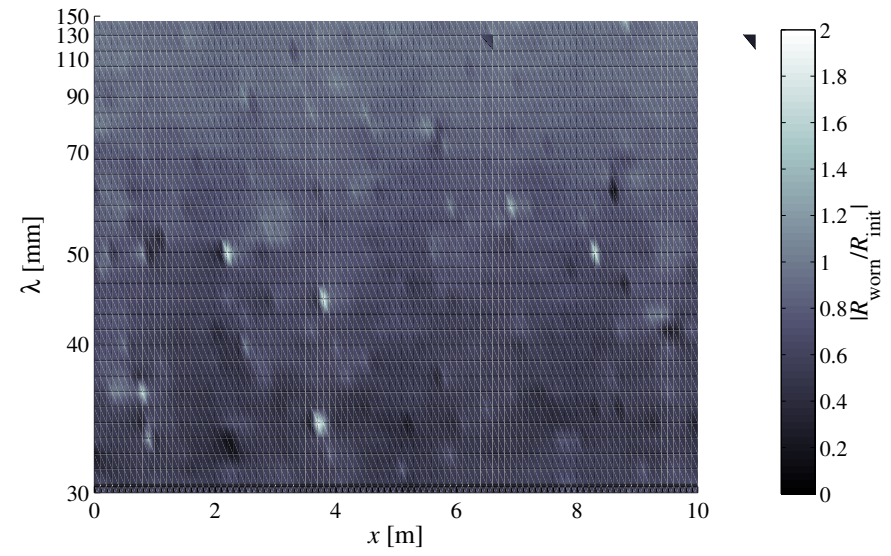

Figure 10. Magnitude spectrum of the quotient between the predicted and initial rail irregularities after 400 wheel passages (corresponding to four simulations of dynamic vehicle-track interaction). The high rail contact of the trailing wheelset is considered using the non-Hertzian and non-steady contact model. Initial rail roughness with magnitude according to the limit in ISO3095 [33].

Curve radius $120 \mathrm{~m}$, vehicle speed $25 \mathrm{~km} / \mathrm{h}$ and friction coefficient 0.6

is negotiating a $120 \mathrm{~m}$ radius curve, large relative lateral wheel-rail displacements are developed at the leading wheelset of each bogie causing the high rail contact to be located towards the gauge corner and gauge face of the rail. For the curve studied in [2], the wear generated by this contact is reduced by the application of lubrication on the high rail. In the simulations, this is considered by modelling a friction coefficient of 0.1 on the gauge face of the high rail. The trailing wheelset of the bogie maintains a close to radial position but is displaced laterally towards the low rail. This means the high rail contact is located on the rail crown. The transfer function $\bar{H}$ calculated for the high rail contact of the trailing wheelset and the low rail contact of the leading wheelset for different friction coefficients are compared in Figure 9. Only results for the non-Hertzian and non-steady contact model are shown. A change in friction coefficient is observed to largely influence both the magnitude and phase of the transfer function. For friction coefficient 0.6 , a peak appears at a wavelength of about $5.2 \mathrm{~cm}$ (corresponding to excitation frequency $126 \mathrm{~Hz}$ at vehicle speed $25 \mathrm{~km} / \mathrm{h}$ ), see Figure 9(a). This peak is not observed for friction coefficient 0.3 . The increase in friction coefficient creates a significant increase in phase at and around the approximate wavelengths $5.2 \mathrm{~cm}$ and $11 \mathrm{~cm}$ (indicating a possibility for roughness growth). This confirms the observations made for the single wavelength irregularities in Section 5.1. Small phase magnitudes are calculated for the high rail contact in the entire investigated wavelength interval, see Figure 9(b). This indicates that corrugation will not grow on the high rail.

\subsection{Simulation of long-term rail roughness growth}

In the previous section, phase shifts between the calculated wear depth and the initial rail irregularity in the order of $\pi / 2$ were found. To conclude if this implies roughness growth, the rail surface needs to be updated in order to predict the long-term change in rail roughness. When the vehicle negotiates the small radius curve, large magnitudes of steady-state lateral and longitudinal creepages and wear are developed at the contacts of the leading and trailing wheelsets, respectively. Since this leads to a uniform wear that does not influence the potential growth of roughness, only the

(a)

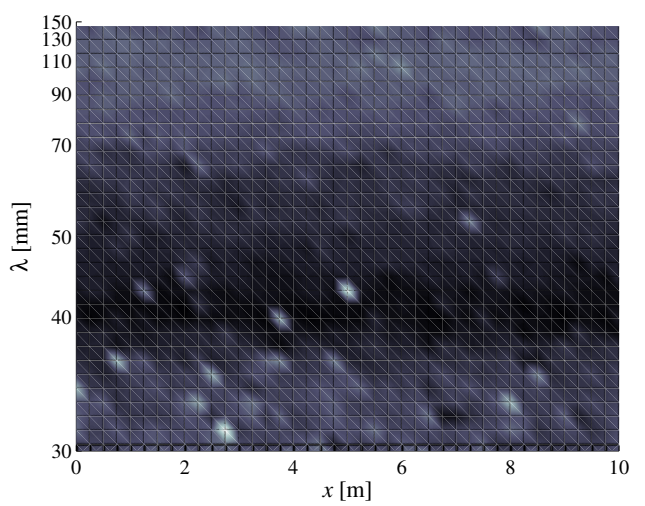

(b)

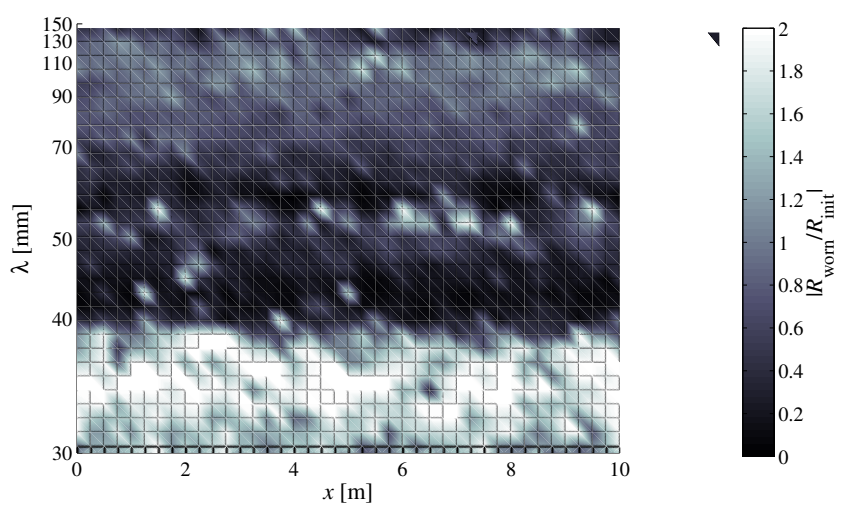

Figure 11. Magnitude spectrum of the quotient between the predicted and initial rail irregularities after 400 wheel passages (corresponding to four simulations of dynamic vehicle-track interaction). Roughness growth is indicated by bright areas. The low rail contact of the leading wheelset is considered using the non-Hertzian and non-steady contact model. Initial rail roughness with magnitude according to with the limit in ISO3095 [33]. Curve radius $120 \mathrm{~m}$ and vehicle speed $25 \mathrm{~km} / \mathrm{h}$. (a) $\mu=0.3$, (b) $\mu=0.6$ 
dynamic part of the wear is considered in the following. Further, since the calculations of dynamic vehicle-track interaction are time consuming, the wear depth caused by one wheel passage is multiplied with the magnification factor 100. The total wear depth for one wheel passage is in the order of $1 \mu \mathrm{m}$ at friction coefficient 0.3 . In the field, variations in for example friction conditions, vehicle speed and wheel profile wear will lead to a distribution of wheel-rail contact positions over the rail head. To account for this, a smoothing procedure is used where the magnified wear depth calculated for one wheel passage is uniformly distributed in the lateral direction of the rail head.

The influence of friction coefficient on the development of rail roughness on the low rail of a $120 \mathrm{~m}$ radius curve is investigated. Vehicle speed $25 \mathrm{~km} / \mathrm{h}$ and 400 wheel passages (corresponding to four simulations of dynamic vehicletrack interaction) are considered. The quotient between the worn and initial rail irregularity is presented in Figure 11. The same initial irregularity was modelled on the low rail for both friction coefficients. In order to investigate the distribution of rail wear in the travelling direction of the vehicle, the rail irregularity spectrum was evaluated in longitudinal steps of $0.1 \mathrm{~m}$. Each spectrum was calculated from 8192 samples corresponding to a $2.3 \mathrm{~m}$ section of rail. A Hanning window was applied to reduce spectral leakage. The proposed simulation model is valid in the frequency range below $200 \mathrm{~Hz}$ and therefore the calculated wear depth was low-pass filtered eliminating wavelengths shorter than $3 \mathrm{~cm}$. For friction coefficient 0.3 , the increasing number of wheel passages leads to a reduction in roughness magnitudes in all of the studied wavelength interval, see Figure 11(a). However for friction coefficient 0.6, it is observed that corrugation growth is predicted at several wavelengths in scattered positions along the rail. The roughness magnitudes after 400 wheel passages (vehicle speed $25 \mathrm{~km} / \mathrm{h}$ and friction coefficient 0.6 ) on the high rail contact of the trailing wheelset are shown in Figure 10. Decreasing roughness magnitudes are observed in the entire studied wavelength interval.

In Figure 11(b), the wear calculated for friction coefficient 0.6 and vehicle speed $25 \mathrm{~km} / \mathrm{h}$ shows roughness growth at wavelength $5.2 \mathrm{~cm}$ at scattered locations along the simulated rail section (see for example at longitudinal coordinate $7 \mathrm{~m}$ ). The potential for roughness development at this wavelength is further investigated by introducing a sinusoidal irregularity of the corresponding wavelength and of constant amplitude in the lateral direction of the low rail. The results for friction coefficient 0.3 and 300 wheel passages, presented in Figure 12(a), show that the peaks of the irregularity are gradually worn down with an increasing number of wheel passages. This is due to the small phase between the calculated wear depth and the present rail irregularity as discussed earlier, see Section 5.1. It was observed in Figure 9(b) that a change in friction coefficient from 0.3 to 0.6 resulted in an average phase of magnitude slightly below $\pi / 2$ at wavelength $5.2 \mathrm{~cm}$. This leads to a translation of the rail irregularity along the rail, see Figure 12(b). Due to the phase delay between the calculated wear and the initial rail irregularity, the translation direction is opposite to the travelling direction of the vehicle.

For an initial single wavelength rail irregularity that was predicted to gradually wear off, the numerical study by Jin et al. [13] showed a simultaneous translation of the corrugation profile. Predictions of long-term roughness development for an initial single wavelength rail irregularity have also been presented by Matsumoto et al. [9]. The increasing corrugation magnitudes were found to involve a longitudinal translation of the corrugation profile. The translation of corrugation peaks demonstrated in the present paper is a similar effect as found in references [9,13]. However, to the authors' knowledge, this is an effect that has not yet been verified by field measurements.

In a previous measurement campaign, the roughness level on the low rail of a $120 \mathrm{~m}$ radius metro curve was observed

(a)

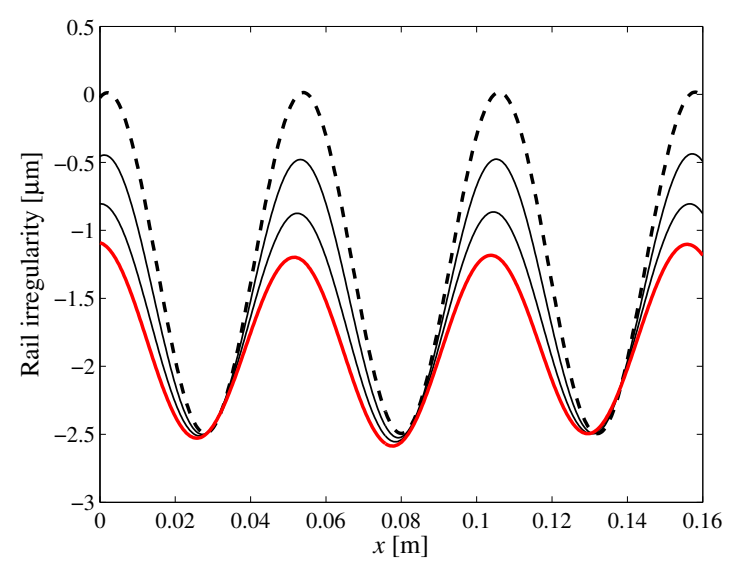

(b)

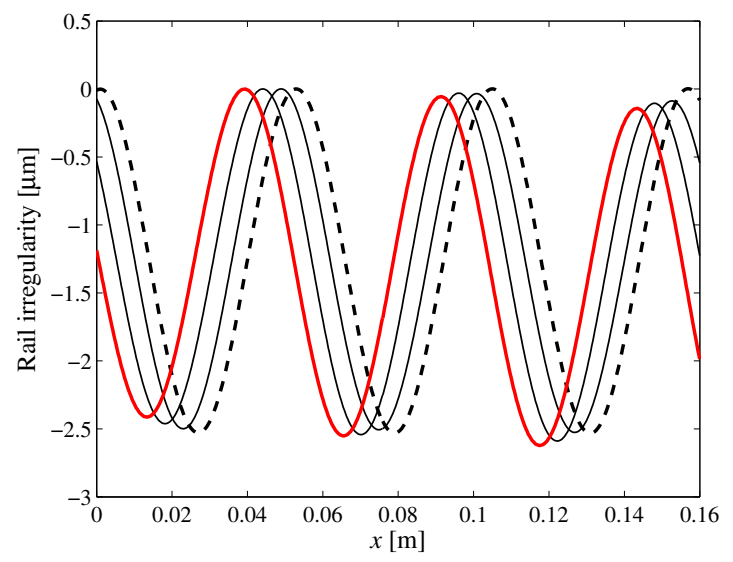

Figure 12. Development of rail roughness after 300 wheel passages (corresponding to three simulations of dynamic vehicle-track interaction). Initial rail irregularity (thick dashed curve), final rail irregularity (thick red solid curve). The low rail contact of the leading wheelset is considered. All results were calculated using the non-Hertzian and non-steady contact model. Initial sinusoidal irregularity of wavelength $5 \mathrm{~cm}$ and with amplitude according to the limit in ISO3095 [33]. Curve radius $120 \mathrm{~m}$ and vehicle speed $25 \mathrm{~km} / \mathrm{h}$. (a) $\mu=0.3$, (b) $\mu=0.6$ 
(a)

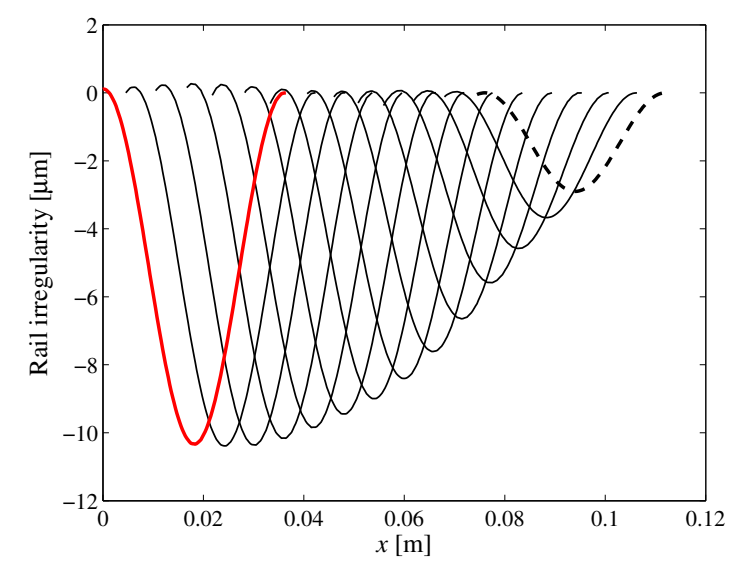

(b)

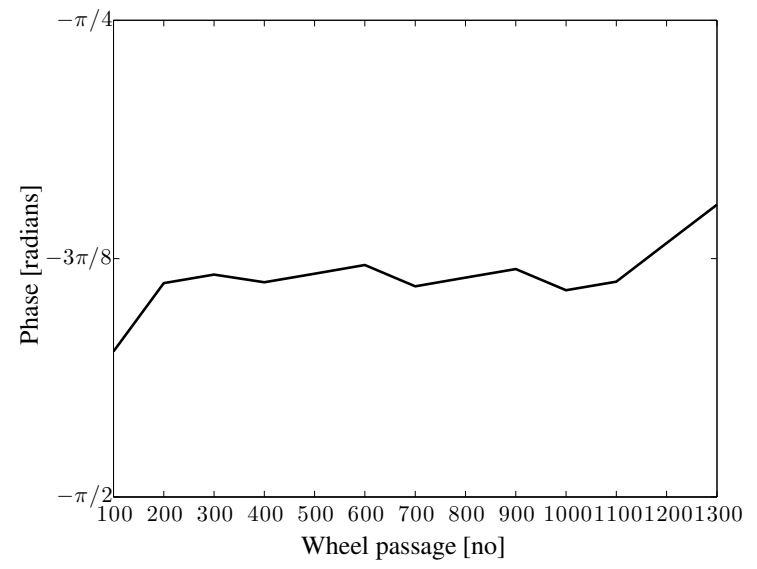

Figure 13. (a) Development of rail roughness after 1200 wheel passages (corresponding to 12 simulations of dynamic vehicle-track interaction) shown for one single wavelength. Initial rail irregularity (thick dashed curve), final rail irregularity (thick red solid curve). (b) Phase between the calculated wear depth and the present rail irregularity at each wheel passage. The low rail contact of

the leading wheelset is considered. All results were calculated using the non-Hertzian and non-steady contact model. Initial sinusoidal irregularity of wavelength $3.8 \mathrm{~cm}$ with amplitude according to the limit in ISO3095 [33]. Curve radius $120 \mathrm{~m}$, vehicle speed $25 \mathrm{~km} / \mathrm{h}$ and friction coefficient 0.6

to increase until approximately 300 days after grinding [2]. Thereafter, additional growth of roughness was moderate. The question of what determines the limit in amplitude of corrugation growth is of fundamental importance. For friction coefficient 0.6, Figure 11(b) shows substantial roughness growth at wavelength $3.8 \mathrm{~cm}$ corresponding to the approximate frequency $183 \mathrm{~Hz}(f=v / \lambda)$. This is caused by a coupled vehicle-track vibration primarily determined by the first anti-symmetric eigenmode of the wheelset. An investigation of the development of rail roughness for 1200 wheel passages on an initial sinusoidal rail irregularity of this wavelength is shown in Figure 13. To clarify the presentation, Figure 13(a) shows the result for a length of rail corresponding to one selected wavelength of the initial rail irregularity. The substantial growth of roughness observed initially is seen to gradually slow down. As shown in Figure 13(b), this is caused by a decreasing phase between the calculated wear depth and the present rail irregularity. After 1200 wheel passages the trough of the rail irregularity is seen to primarily move backwards with a constant amplitude.

\section{CONCLUDING REMARKS}

A time-domain model for the prediction of long-term rail roughness growth on small radius curves has been presented. The present model captures the dynamic vehicle-track interaction in a broad frequency range (up to at least $200 \mathrm{~Hz}$ ). Both low-frequency dynamics of the full vehicle due to curving and high-frequency vehicle-track vibration due to short-wavelength rail roughness excitation are accounted for. The structural flexibility of the rails and wheelset axles are included by application of the finite element method. The influence of non-Hertzian and non-steady effects in the wheel-rail contact model on rail wear is considered by employing the variational method by Kalker implemented in a post-processing step. To calculate an accurate location of the contact, the three-dimensional irregular surfaces of the wheel and rail are considered in each time-step in the simulation of dynamic vehicle-track interaction. This allows for the contact detection problem, used in combination with Hertz/FASTSIM, to account for the wheelset yaw angle as well as for the surface irregularities and structural flexibilities of wheelset and rail.

The implementation of the non-Hertzian and non-steady contact model has been verified versus Hertz/FASTSIM for a case of cylindrical wheel and rail profiles and contact conditions involving partial slip. The phase between the wear and the present rail irregularity calculated with Hertz/FASTSIM in combination with pre-calculated contact point functions (KPF) to solve the contact detection problem was significantly over-estimated compared to that obtained from the nonHertzian and non-steady contact model. Although particularly legible at short wavelengths (below approximately three times the longitudinal length of the contact area), significant differences were observed also at longer wavelengths (around $10 \mathrm{~cm}$ ). When the contact positions used in conjunction with Hertz/FASTSIM were updated by applying the refined contact detection algorithm, the calculated phase was similar to the non-Hertzian and non-steady contact model in the entire studied wavelength interval. This emphasises the importance of the contact detection problem in calculations of long-term roughness development.

The development of roughness on the low rail of the $120 \mathrm{~m}$ radius curve was found to be influenced by the level of friction. For friction coefficient 0.3 , the prediction of long-term roughness development showed decreasing magnitudes in the entire studied wavelength interval. The corresponding calculation for friction coefficient 0.6 indicated corrugation 
development at several wavelengths. Calculations of long-term roughness development on the high rail contact for the trailing wheelset indicated no corrugation growth. This agrees with the observations in [2]. For all cases the gauge face of the high rail was modelled with friction coefficient 0.1 (lubrication).

The question of what determines a limit for the roughness magnitude for fully grown corrugation was investigated for an initial sinusoidal irregularity of wavelength $3.8 \mathrm{~cm}$. Due to the gradual decrease in phase between the calculated wear depth and the present rail irregularity for an increasing number of wheel passages, the rapid roughness growth observed initially is eventually stopped at approximately five times its initial amplitude. At this stage of roughness development, the rail irregularity is seen to primarily move backwards with a constant amplitude. This is a non-linear effect that cannot be investigated by only assessing the wear depth magnitude. The authors emphasise the verification of these results towards field measurements as an important part of future work.

Recent models accounting for several wavelength-fixing mechanisms and non-Hertzian and non-steady contact models for the wheel-rail contact have been unable to predict corrugation growth [11-14]. Apart from indicating the significant influence of the contact model, this has created a discussion about still missing components in the model to reach a comprehensive explanation of the development of corrugation. With regard to this, the current work underlines the importance of the phase between the calculated wear depth and the present rail irregularity. For example at wavelength $3.8 \mathrm{~cm}$, peaks in the wear depth were found for friction coefficients 0.3 and 0.6 as well as for both the low and high rail contacts. However, due to the associated phase between the calculated wear depth and the present rail irregularity, it was only for the low rail contact of the leading wheelset and for friction coefficient 0.6 that corrugation was generated. Corrugation for the other cases was ruled out.

\section{ACKNOWLEDGEMENTS}

This work was performed as part of the activities within the Centre of Excellence CHARMEC (CHAlmers Railway MEChanics). Discussions with Dr Anders Frid of Bombardier Transportation Sweden are acknowledged.

\section{REFERENCES}

[1] S.L. Grassie, J. Kalousek, Rail corrugation: characteristics, causes and treatments. Proceedings of the Institution of Mechanical Engineers, Part F: Journal of Rail and Rapid Transit 207 (1993) 57-68

[2] P.T. Torstensson, J.C.O. Nielsen, Monitoring of rail corrugation growth due to irregular wear on a railway metro curve. Wear 267 (2009) 556-561

[3] K. Hempelmann, F. Hiss, K. Knothe, B. Ripke, The formation of wear patterns on rail tread. Wear 144 (1991) 179195

[4] K. Hempelmann, K. Knothe, An extended linear model for the prediction of short pitch corrugation. Wear 191 (1996) 161-169

[5] A. Igeland, H. Ilias, Rail head corrugation growth predictions based on non-linear high frequency vehicle/track interaction. Wear 213 (1997) 90-97

[6] J.C.O. Nielsen, High-frequency vertical wheel-rail contact forces—validation of a prediction model by field testing. Wear 265 (2008) 1465-1471

[7] P.T. Torstensson, J.C.O. Nielsen, L. Baeza, Dynamic train-track interaction at high vehicle speeds - modelling of wheelset dynamics and wheel rotation. Journal of Sound and Vibration 330 (2011) 5309-5321

[8] C. Andersson, A. Johansson, Prediction of rail corrugation generated by three-dimensional wheel-rail interaction. Wear 257 (2004) 423-434

[9] A. Matsumoto, Y. Sato, H. Ono, M. Tanimoto, Y. Oka, E. Miyauchi, Formation mechanism and countermeasures of rail corrugation on curved track. Wear 253 (2002) 178-184

[10] B. Kurzeck, Combined friction induced oscillations of wheelset and track during the curving of metros and their influence on corrugation. Wear 271 (2011) 299-310

[11] G. Xie, S.D. Iwnicki, Calculation of wear on a corrugated rail using a three-dimensional contact model. Wear 265 (2008) 1238-1248

[12] G. Xie, S.D. Iwnicki, Simulation of wear on a rough rail using a time-domain wheel-track interaction model. Wear 265 (2008) 1572-1583

[13] X.S. Jin, Z.F. Wen, K.Y. Wang, Z.R. Zhou, Q.Y. Liu, C.H. Li, Three-dimensional train-track model for study of rail corrugation. Journal of Sound and Vibration 293 (2006) 830-855

[14] B. Croft, C. Jones, D. Thompson, The effect of rail dampers on roughness growth rates with multiple wear mechanisms, non-Hertzian contact and velocity dependent friction. Proceedings of the 8th International Conference on Contact Mechanics and Wear of Rail/Wheel Systems (CM2009), Firenze, Italy, September (2009) 15-18

[15] G. Xie, S.D. Iwnicki, A rail roughness growth model for a wheelset with non-steady, non-Hertzian contact. Vehicle System Dynamics 48 (2010) 1135-1154

[16] S.L. Grassie, Rail corrugation: advances in measurement, understanding and treatment. Wear 258 (2005) 1224- 
1234

[17] P.T. Torstensson, J.C.O. Nielsen, Simulation of dynamic vehicle-track interaction on small radius curves. Vehicle System Dynamics 49 (2010) 1711-1732

[18] A. Pieringer, Time-domain modelling of high-frequency wheel/rail interaction. Ph. D. Dissertation, Department of Civil and Environmental Engineering, Chalmers University of Technology, Göteborg, Sweden (2011)

[19] J.F. Archard, Contact and rubbing of flat surfaces. Journal of Applied Physics 24 (1953) 981-988

[20] DEsolver, GENSYS User's manual. (2007) www.gensys.se

[21] S.L. Grassie, R.W. Gregory, D. Harrison, K.L. Johnson, The dynamic response of railway track to high frequency vertical excitation. Journal of Mechanical Engineering Science 24 (1982) 77-90

[22] C. Andersson, Modelling and simulation of train-track interaction including wear prediction. Ph. D. Dissertation, Department of Applied Mechanics, Chalmers University of Technology, Göteborg, Sweden (2003)

[23] K. Popp, H. Kruse, I. Kaiser, Vehicle-track dynamics in the mid-frequency range. Vehicle System Dynamics 31 (1999) 423-464

[24] A.A. Shabana, Dynamics of multibody systems. (2005) pp. 374

[25] R.D. Cook, D.S. Malkus, M.E. Plesha, Concepts and applications of finite element analysis. Third edition, John Wiley \& Sons, New York (1989) pp. 630

[26] M. Hiensch, J.C.O. Nielsen, E. Verheijen, Rail corrugation in the Netherlands - measurements and simulations. Wear 253 (2002) 140-149

[27] K.L. Johnson, Contact mechanics. Cambridge University Press, New York (1985) pp. 468

[28] J.J. Kalker, Three-dimensional elastic bodies in rolling contact. Kluwer Academic Publishers, Dordrecht, Boston, London (1990) pp. 314

[29] K. Knothe, A. Groß-Thebing, Short wavelength rail corrugation and non-steady-state contact mechanics. Vehicle System Dynamics 46 (2008) 49-66

[30] J.F. Archard, W. Hirst, The wear of metals under unlubricated conditions. Proceedings of the Royal Society of London. Series A. Mathematical and Physical Sciences 236 (1956) 397-410

[31] U. Olofsson, T. Telliskivi, Wear, plastic deformation and friction of two rail steels - a full-scale test and a laboratory study. Wear 254 (2003) 80-93

[32] R. Nilsson, On wear in rolling/sliding contacts. Ph. D. Dissertation, Department of Machine Design, Royal Institute of Technology, Stockholm, Sweden (2005)

[33] EN ISO 3095:2005, Railway applications - acoustics - measurements of noise emitted by railbound vehicles. 


\section{APPENDIX}

\section{Rail}

The rail parameters in Table 1 are used in the Euler-Bernoulli-Saint-Venant beam theory. The rail inclination is 1:40.

Table 1: Parameters of the rail model (BV50 profile)

\begin{tabular}{l|c}
\hline Parameter & Value \\
\hline Bending stiffness about lateral axis $E I_{\mathrm{Y}}\left[\mathrm{MNm}^{2}\right]$ & 4.32 \\
\hline Bending stiffness about vertical axis $E I_{\mathrm{Z}}\left[\mathrm{MNm}^{2}\right]$ & 0.74 \\
\hline Saint-Venant torsional stiffness $G K_{\mathrm{V}}\left[\mathrm{MNm}^{2}\right]$ & 0.18 \\
\hline Mass distribution $[\mathrm{kg} / \mathrm{m}]$ & 50 \\
\hline Cross-section area $\left[\mathrm{m}^{2}\right]$ & $6.371 \cdot 10^{-3}$ \\
\hline Young's modulus $\left[\mathrm{GN} / \mathrm{m}^{2}\right]$ & 210 \\
\hline Poisson's ratio & 0.3 \\
\hline
\end{tabular}

\section{Track support}

The track support parameters given in Table 2 were determined by a tuning of the model to obtain good agreement between calculated and measured receptances.

Table 2: Parameters of the track support model. Each parameter may hold values which correspond to the translational directions $x$ (longitudinal), $y$ (lateral) and $z$ (vertical), and three values which correspond to the rotations $\varphi_{i}(i=x, y, z)$. A dash (-) means that the corresponding degree-of-freedom is constrained to zero

\begin{tabular}{l|c|c|c|c|c|c}
\hline Parameter & $x$ & $y$ & $z$ & $\varphi_{x}$ & $\varphi_{y}$ & $\varphi_{z}$ \\
\hline $\mathbf{k}_{\mathrm{p}}[\mathrm{MN} / \mathrm{m}]$ & - & 30 & 200 & 300 & 469 & 260 \\
\hline $\mathbf{k}_{\mathbf{b}}[\mathrm{MN} / \mathrm{m}]$ & - & 5 & 30 & - & - & - \\
\hline $\mathbf{c}_{\mathrm{p}}[\mathrm{kNs} / \mathrm{m}]$ & - & 5 & 48 & 100 & 128 & 128 \\
\hline $\mathbf{c}_{\mathbf{b}}[\mathrm{kNs} / \mathrm{m}]$ & - & 32 & 32 & - & - & - \\
\hline $\mathbf{m}_{\mathbf{s}}[\mathrm{kg}]$ & - & 125 & 125 & - & - & - \\
\hline
\end{tabular}

